\title{
Rayleigh Friction, Newtonian Cooling, and the Linear Response to Steady Tropical Heating*
}

\author{
Zhaohua Wu, David S. Battisti, and E. S. Sarachik \\ Department of Atmospheric Sciences, University of Washington, Seattle, Washington
}

(Manuscript received 1 May 1998, in final form 19 August 1999)

\begin{abstract}
A series of studies are performed to examine the response of the tropical atmosphere to a prescribed steady, large-scale, elevated heat source (i.e., a region of persistent precipitation). Special emphasis is placed on the surface wind response in two idealized cases in which dissipation is achieved exclusively by Rayleigh friction or by Newtonian cooling. Starting from the linearized equations on an equatorial beta plane, theoretical arguments are presented that suggest there are qualitative differences in the solutions of these two models. A dry spectral primitive equation model of the atmosphere is employed and confirms the results obtained from the analytical studies.

The results from both the analytical study and the numerical simulations are consistent in showing that Rayleigh friction and Newtonian cooling play totally different roles in the tropical atmosphere. Newtonian cooling homogenizes the atmospheric motion in the vertical direction, and a strong, vertically uniform wind is found below the base of the heat source. When Rayleigh friction dominates, the circulation driven by the heat source is confined to the layer where the heat source is located. It is also shown that a strong Hadley circulation is associated with reasonable strong Rayleigh friction, but not with Newtonian cooling alone.

Finally, the numerical solution is found for the case where Newtonian cooling acts uniformly in the vertical and Rayleigh friction is included in the lower atmosphere to mimic crudely the dissipation of momentum in the boundary layer. The introduction of the simple boundary layer dramatically reduces the surface circulation that was supported in the Newtonian cooling alone case. Together these results suggest a significant surface circulation is unlikely to be driven by an elevated heat source if it resides above the top of the boundary layer.
\end{abstract}

\section{Introduction}

The distribution of tropical heating is fundamental to the large-scale circulation of the tropical atmosphere. Tropical heating arises from the latent heat released in collections of deep precipitating cumulonimbus clouds, forming at cloud base (approximately $600 \mathrm{~m}$ ) and ending at the tropospause (at $16 \mathrm{~km}$ ). In the western Pacific, the heat sources, averaged over periods longer than a month, are organized in horizontally globular shapes, while in the interior of the tropical Pacific, the sources are more zonally elongated in shape. Tropical heating drives the climatology: the seasonally varying Hadley, Walker, and monsoonal circulations. Interannual variations of tropical heating can be identified with the ENSO phenomenon, which in turn helps drive midlatitude variability on interannual timescales (e.g., Graham et al.

\footnotetext{
* Joint Institute for the Study of the Atmosphere and Ocean Contribution Number 465.
}

Corresponding author address: Dr. Zhaohua Wu, COLA, 4041 Powder Mill Road, Suite 302, Calverton, MD 20706.

E-mail: zhwu@cola.iges.org
1994; Lau and Nath 1994). We see that no explanation of the large-scale tropical circulations, both steady and varying, can be given without describing the circulations due to these heat sources taken interactively. The beginning of this process is considering the circulations driven by a single heat source. While recognizing the existence of more rapid fluctuations, we will confine ourselves to very slowly varying large-scale heat sources and the circulations driven by these heat sources.

The moisture budget in the Tropics indicates that the precipitation in the heat source regions is fed by convergence in the lowest few kilometers of the atmosphere: only at low levels is the specific moisture large enough to allow the convergence to maintain rainfall of order of many millimeters a day. It is diagnostically true that the regions of persistent low-level convergence (upward motion) are relatively small compared to the large regions of persistent divergence (downward motion). The question naturally arises, what is the cause of this low-level moisture convergence? Is it caused by the circulations driven by the thermal sources themselves or is it driven by separate low-level processes disconnected from the circulations driven by the thermal sources? If the first case is applicable, then the circulations driven by the thermal sources would have to be just the 
right strength to maintain the sources: too little and the sources would decay, too much and a growing instability (the so-called conditional instability of the second kind, or CISK) would arise. In the second case, the upperlevel circulations would be driven by the thermal sources, but the locations and strength of the sources would be due to low-level processes (see, e.g., Battisti et al. 1989). It is of course possible that a mixed case would apply: the low-level convergence is mostly due to one process but modified by the other (Schneider and Lindzen 1977).

In this paper we will study the problem of thermally driven circulations (especially the low-level winds) in the Tropics by examining the responses to a single isolated steady heat source in the presence of different combinations of thermal damping and momentum damping. We will follow the methodology of Geisler (1981), who specified the magnitude and vertical and horizontal shape of the heat source, prescribed the radiative and frictional parameters, and numerically examined the linear response to this source. We will do something similar but with this difference: we will try to understand why the circulations are what they are and how they depend on the specified frictional and radiative parameters. We will also examine the question posed above (which Geisler did not address): is the lowlevel convergence driven by a thermal source of sufficient amplitude enough to maintain the prescribed heating itself?
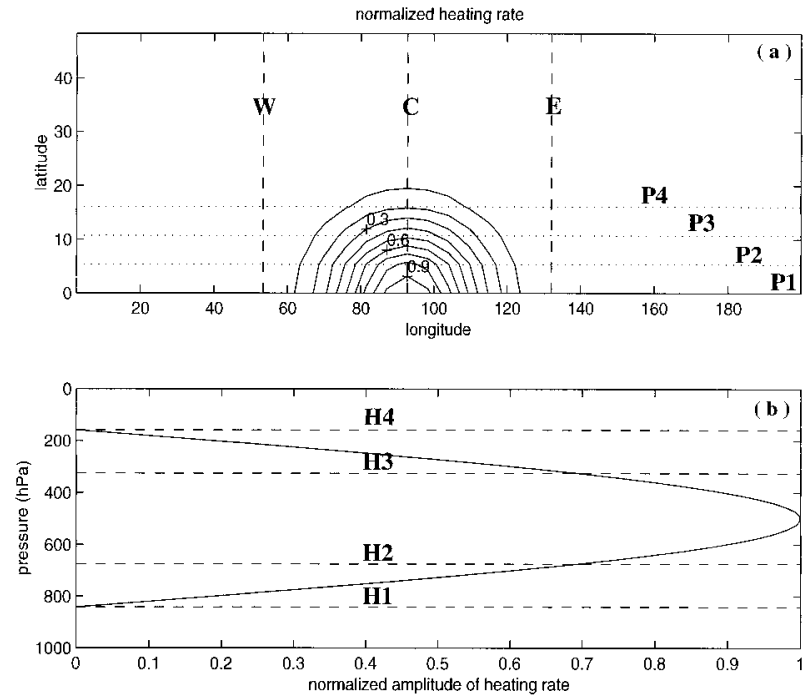

FIG. 1. Distribution of normalized heating rate on the horizontal plane (a) and in the vertical direction (b). In (a), the solid lines represent the normalized heating rate; the dashed lines and the dotted lines show the locations where the physical fields are analyzed later; $\mathrm{W}, \mathrm{C}$, and $\mathrm{E}$ are the longitudes $53.4^{\circ}, 92.8^{\circ}$, and $132.2^{\circ} \mathrm{E}$, respectively; and $\mathrm{P} 1, \mathrm{P} 2, \mathrm{P} 3$, and $\mathrm{P} 4$ are the latitudes $0^{\circ}, 5.4^{\circ}, 10.7^{\circ}$, and $16.1^{\circ} \mathrm{N}$ respectively. In (b), the solid line represents the dependence of heating rate on height; the dashed lines show the heights where the physical fields are analyzed later; $\mathrm{H} 1, \mathrm{H} 2, \mathrm{H} 3$, and $\mathrm{H} 4$ are the altitudes 840 , 675,325 , and $160 \mathrm{hPa}$, respectively.
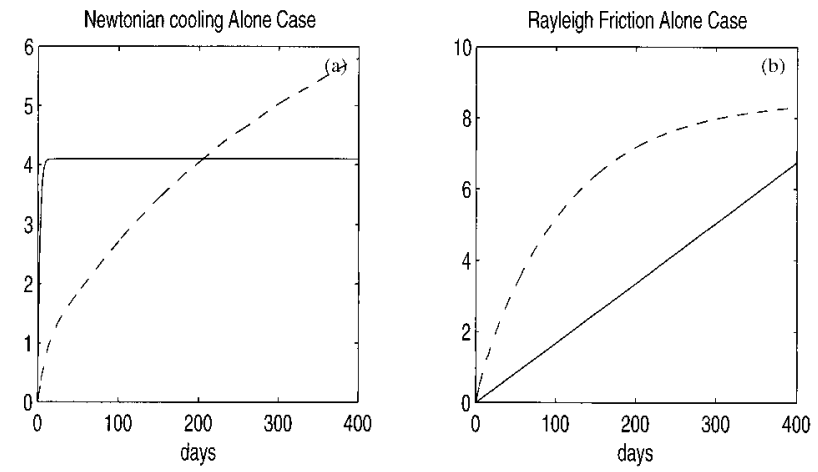

FIG. 2. Time evolution of global potential energy (solid lines) and global kinetic energy (dashed lines) (a) for the Newtonian cooling alone case and (b) for the Rayleigh friction alone case. The units for all plots except the potential energy in the Rayleigh friction alone case are $10^{18} \mathrm{~J}$. For the potential energy in the Rayleigh friction alone case, the units are $10^{20} \mathrm{~J}$.

A number of papers in the literature are highly relevant to this inquiry. The simple thermal source model of Gill (1980) assumed that at low frequency, the observed heating resulting from large-scale cumulus convection in the Tropics could be grossly represented by a single (sinusoidal) mode in the vertical, with the forced response taking the shape (or its derivative) of this mode as well. The horizontal structure of the analytical solutions of Gill are very similar to the numerical results from a two-layer model designed by Webster (1972), and Webster also interpreted his external forcing as the simplification of large-scale cumulus heating. The relationship of convergence to heating is automatically built into this model by the artifice of specifying a single mode: the heating is artificially brought right to the surface. In this case the question of the propagation of the forced signals from the bottom of the heat source to the surface therefore cannot be considered.

There are a number of papers that assume that lowlevel convergence is produced by hydrostatic pressure forces induced by sea surface temperature (SST) gradients and therefore that in the absence of feedback from the thermally forced circulation, the location and strength of the thermal forcing would be determined solely by boundary layer processes interacting with SST. The first and most influential among these papers was Lindzen and Nigam (1987). In their model, the surface convergence is everywhere rapidly vented by convection in the presence of the strong thermal damping. Also, the boundary layer anchors the location of convection to the underlying SST anomalies and the upper-level convection acts primarily as a reservoir for the loweratmosphere heat and perturbations. The lack of feedback of the thermal source to surface circulations was indicated in classic thermal forcing papers by Schneider and Lindzen (1977) and Schneider (1977), where specified zonally symmetric forcing, characteristic of the Hadley circulation, fails to modify the low-level convergences produced by surface temperature gradient. 


\section{Newtonian-Cooling-Alone-Case}
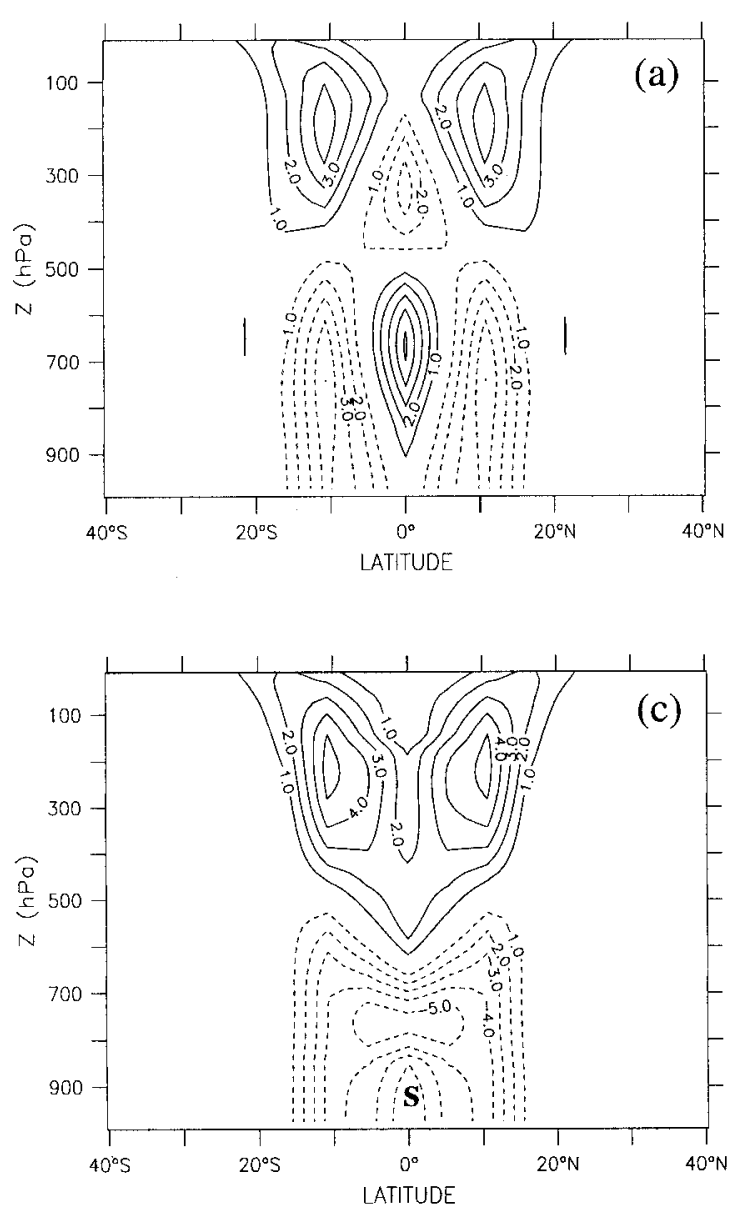

While the Lindzen and Nigam model simulates the surface wind anomalies in the tropical eastern Pacific with some success, the model is incapable of explaining the strong surface wind anomalies that are observed in some regions where the SST gradients are very small in the tropical Pacific. Observational results from Deser and Wallace (1987), Deser and Wallace (1990), and Mitchell and Wallace (1996) showed that the July-November outgoing longwave radiation, a proxy for regions of persistent precipitation, and the surface wind are well correlated, especially in the western tropical Pacific where the SST gradient is very small (i.e., less than $0.25 \mathrm{~K}$ per $1000 \mathrm{~km}$ ). Rasmusson and Carpenter (1982) illustrated that in the western tropical Pacific, although there is almost no SST gradient, the monthly climatological surface wind is significant. Geisler, in the paper previously mentioned, showed that a strong surface wind develops in response to a prescribed, elevated heat source. Zebiak (1986), modifying the Gill model's heating to include a CISK-like component based on the surface mass convergence, noted that the convergence

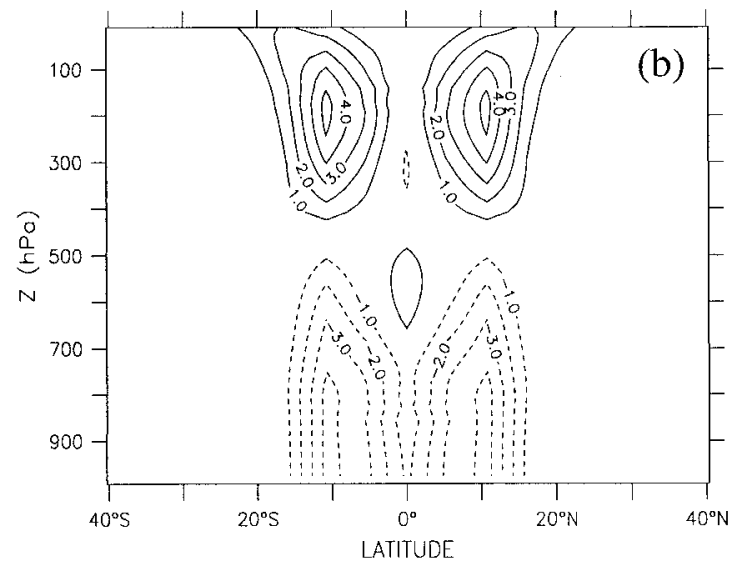

FIG. 3. Zonal velocities at different longitudes for the Newtonian cooling alone case. (a) At $53.4^{\circ} \mathrm{E}$, (b) at $92.8^{\circ} \mathrm{E}$, (c) at $132.2^{\circ} \mathrm{E}$. Westerlies are represented by solid lines, and easterlies are represented by dashed lines. Contour interval is $1.0 \mathrm{~m} \mathrm{~s}^{-1}$ in every panel, and the zero contour lines are not shown. The "s" in (c) represents a minimum center. feedback term improved the amplitude and the pattern of the simulated surface wind response. These studies imply that the large-scale cumulus heating can drive significant surface winds.

To clearly understand the mechanism of how the large-scale cumulus heating drives low-level circulations, one must consider a forced problem in the absence of the SST gradient. Any surface winds forced under this circumstance must have been thermally driven. In order to solve the problem correctly, the propagation characteristic of the thermally forced signals from cloud base to the surface has to be considered, since the problem involves propagation; the results must be shown to exist independent of the existence of an artificial lid imposed at the top, since lids are known to induce artificial standing modes that can induce artificial results throughout a vertical column (Lindzen et al. 1968).

These considerations provide the background for the current idea of the impact of a large-scale elevated heat source on the low-level circulation, which is based mainly on the propagation of energy away from the 


\section{Newtonian-Cooling-Alone-Case}
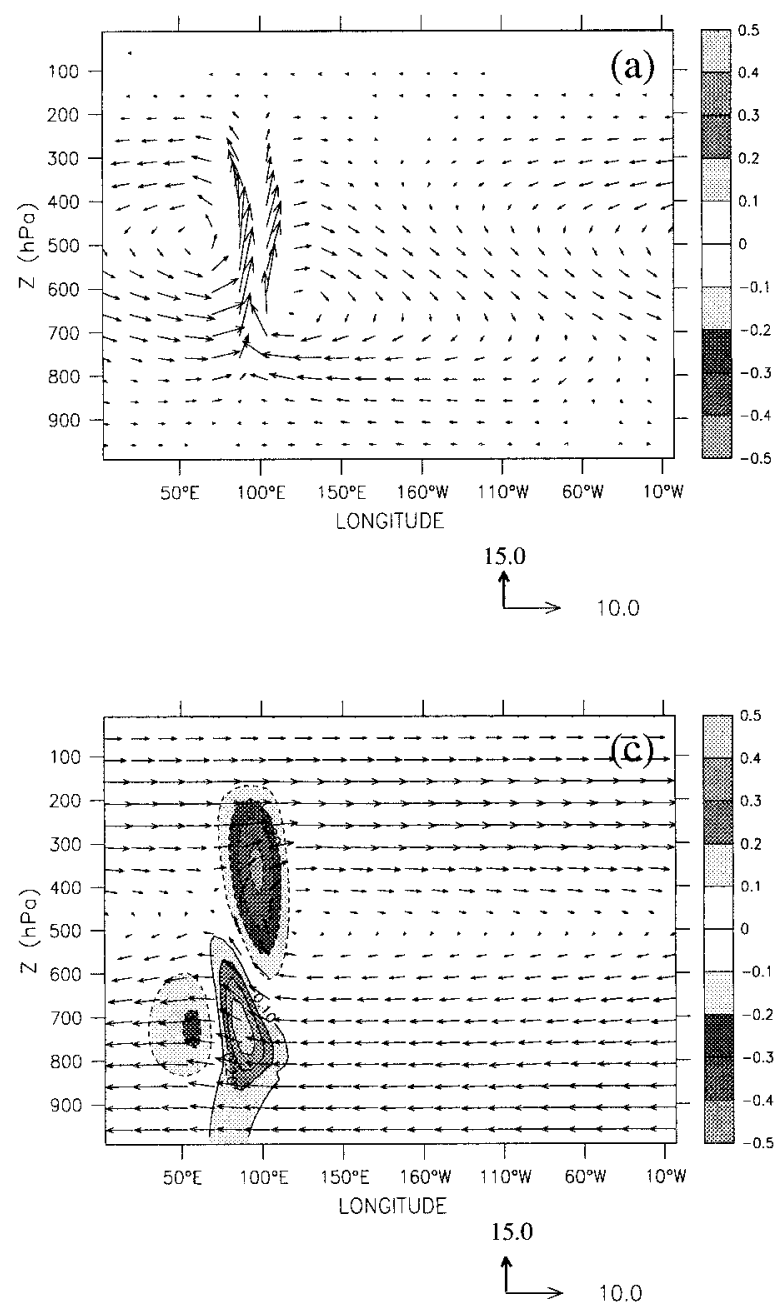
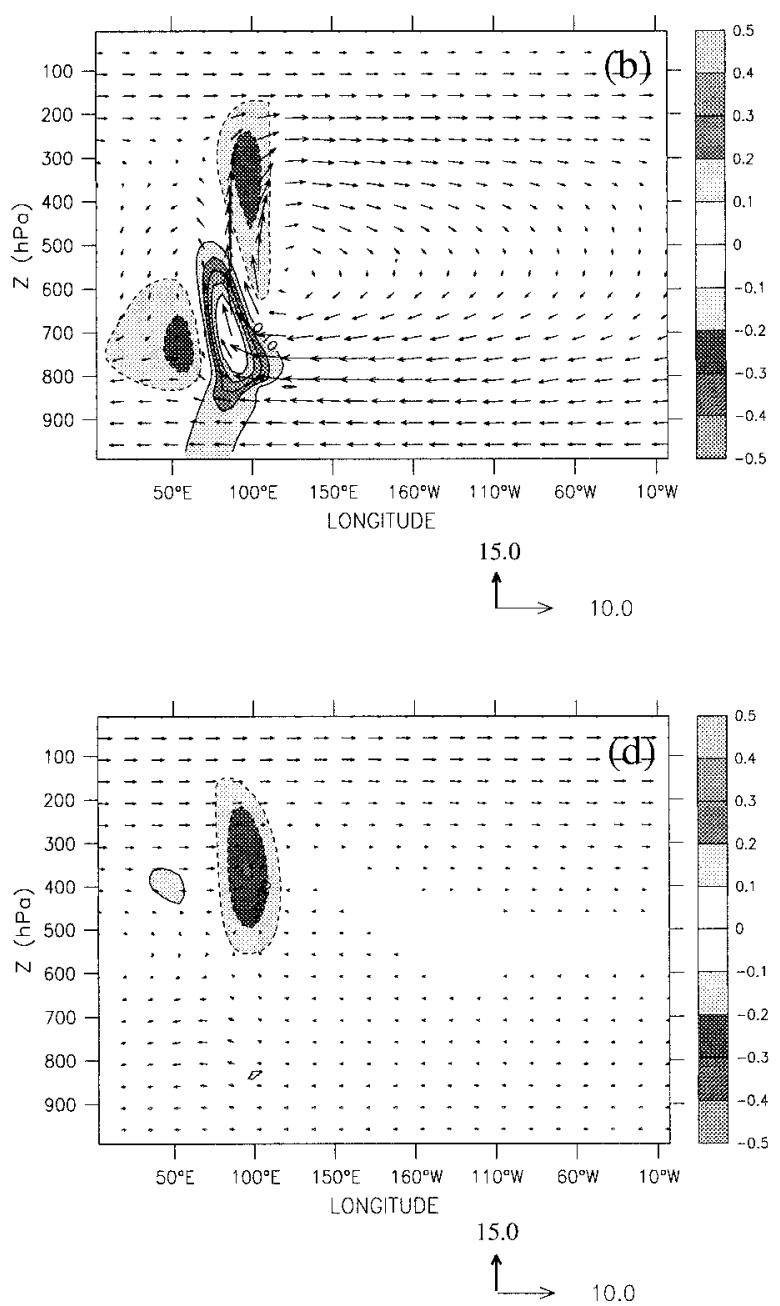

FIG. 4. The zonal overturning circulations at different latitudes for the Newtonian cooling alone case. The vector field of zonal velocity (with unit $\mathrm{m} \mathrm{s}^{-1}$ ) and vertical velocity $\left(-d p / d t\right.$ with unit $\mathrm{hPa}$ day $^{-1}$ ) are superposed on the contoured meridional velocity (with contour interval $0.1 \mathrm{~m} \mathrm{~s}^{-1}$ ). The zonal velocity and the vertical velocity are scaled separately and are shown under each panel, respectively. (a) At the equator, (b) at $5.4^{\circ} \mathrm{N}$, (c) at $11.7^{\circ} \mathrm{N}$, (d) at $16.1^{\circ} \mathrm{N}$.

bottom of the thermal forcing by free waves in a viscous medium. The linear wave theory for the Tropics can be found in the seminal paper by Lindzen (1967). Lindzen solved the three-dimensional linearized equations for an inviscid atmosphere on an equatorial beta plane in a vertically semibounded space, and derived some of the propagation properties of the free and the forced waves. From Lindzen's formulas, it can be shown (Wu et al. 1999) that the vertical group velocity for these equatorial trapped waves is proportional to the third power of the frequency for large-scale motion at low frequencies. This suggests that under low-frequency forcing and moderate dissipation, the vertical energy propagation is so slow that the signal is damped before reaching far from the heat source (the distance of propagation is characterized by the product of the group velocity and the inverse of the damping rate) and therefore never reaches the surface.

This argument is, however, flawed. The solutions given by Lindzen (1967) are based only on part of the meridional eigenfunctions, those with discrete values of positive equivalent depth. ${ }^{1} \mathrm{Wu}$ (1998) and $\mathrm{Wu}$ et al. (1999) point out that the complete set of eigenfunctions resulting from the meridional eigenvalue-eigenfunction

\footnotetext{
${ }^{1}$ We here follow the tradition (Lamb 1932; Lindzen 1967; Philander 1990) and call the eigenvalue, which is determined by the meridional eigenvalue-eigenfunction problem (e.g., Laplace tidal equation on a sphere), the equivalent depth. In such a definition, the equivalent depth does not have the physical meaning of the depth of a shallow water system.
} 


\section{Newtonian-Cooling-Alone-Case}
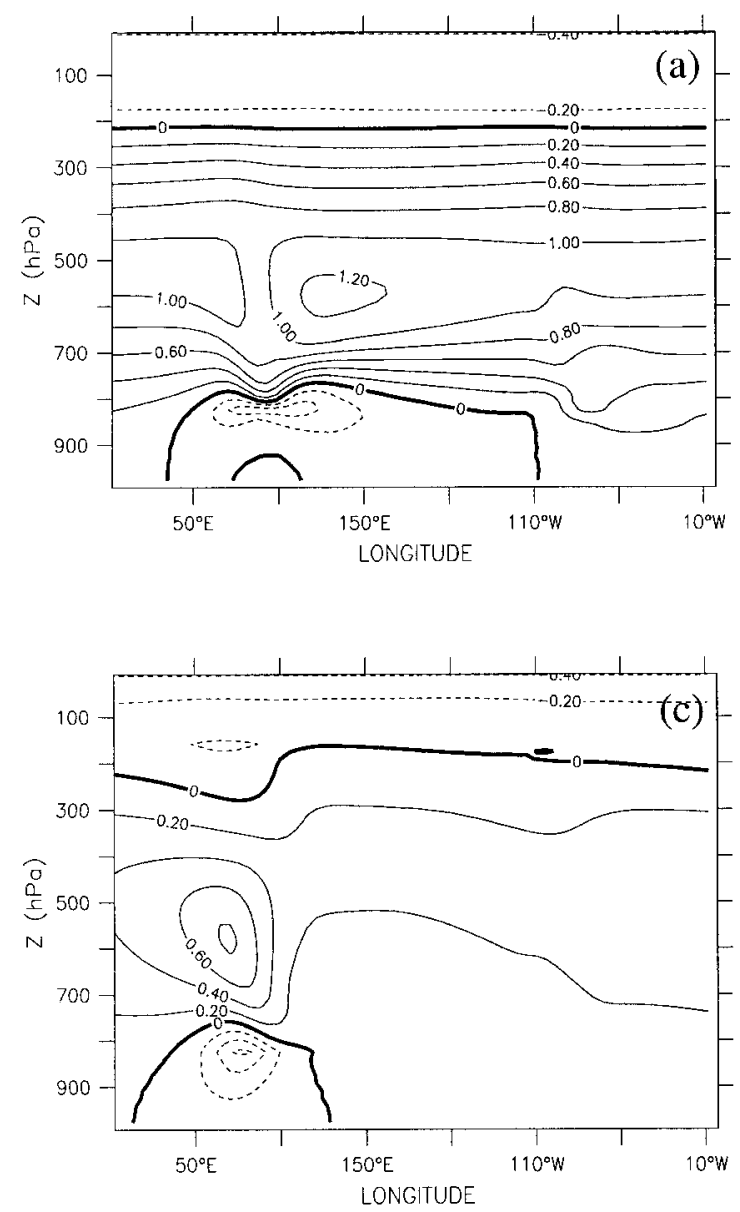

problem $^{2}$ at low frequencies includes three types of eigenfunctions: 1) the eigenfunctions of discrete positive equivalent depth that shrink to an infinitesimal region around the equator as the frequency goes to zero, 2) the eigenfunctions of discrete positive equivalent depth that asymptotically approach two sinusoidal functions (of which one is symmetric and the other is antisymmetric about the equator) before they reach their turning latitudes, and 3) the eigenfunctions of continuous negative equivalent depth that were previously not discovered on an equatorial beta plane but their counterparts do exist on a spherical earth (Flattery 1967, Lindzen 1990). Wu

\footnotetext{
${ }^{2}$ The meridional eigenfunctions are obtained by applying variable separation method on a single equation for meridional velocity derived from the original set of linear equations. The original set of linear equations has one more solution when meridional velocity is zero. For that particular solution, the vertical group velocity of the forced signal is proportional to the square of the frequency. However, when frequency is low, external forcing of large meridional scale does not project much onto that solution.
}

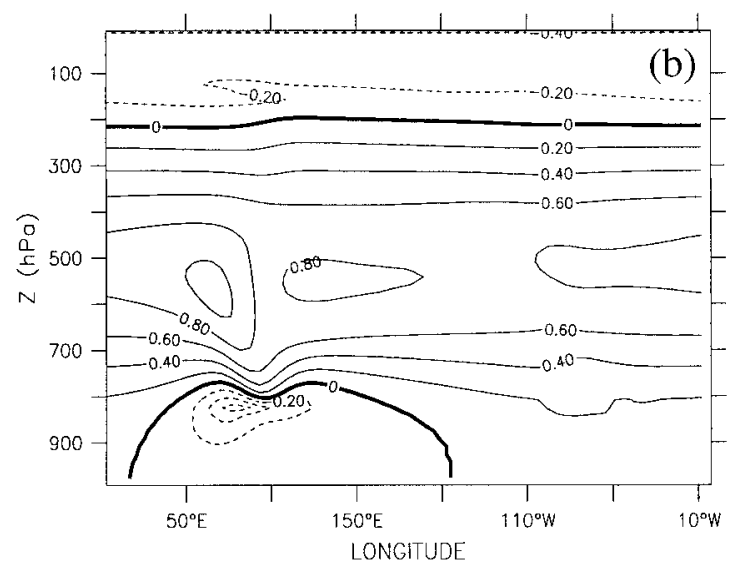

FIG. 5. Temperature perturbations at different latitudes for the Newtonian cooling alone case. (a) At the equator, (b) at $5.4^{\circ} \mathrm{N}$, (c) at $11.7^{\circ} \mathrm{N}$. Contour interval is $0.2^{\circ} \mathrm{C}$ in each panel.

et al. (1999) also show that the third type of the eigenfunctions account for the most of the solution for the large-scale low-frequency forced motions in the tropical atmosphere.

In an atmosphere without any damping, the forced solution associated with the first two types of eigenfunctions can propagate vertically, while the forced response associated with the last type of the eigenfunctions is vertically evanescent. In the presence of damping, the structure of the forced signals is highly dependent on the nature of the damping. Wu et al. (1999) show that when the only damping is linear thermal damping (Newtonian cooling) the response to a largescale low-frequency periodic heat source is qualitatively different from what is expected from the simple group velocity argument. In that case, the large-scale heat source projects its energy mainly onto the modes of which the absolute value of the equivalent depth is large. The solutions to the vertical structure equation give vertically uniform winds below the heat source down to the surface. Hence, the above group velocity argument 
is clearly not applicable when the atmosphere is subjected to linear thermal damping only.

The purpose of this present study is to investigate the linear response of tropical atmosphere to a steady, largescale, elevated heat source in the presence of different types of damping. Emphasis is placed on the low-level circulations, especially the surface winds, driven by the heating in the presence of different types of damping. This part of the study can also be regarded as a numerical extension of the theory developed by Wu et al. (1999) where only Newtonian cooling acts. In section 2, we examine separately the roles of Newtonian cooling and Rayleigh friction, the two simplest damping mechanisms used in the theoretical studies, by analytically obtaining the solutions to a steady thermal source in the presence of either Rayleigh friction or Newtonian cooling. To further support the analytical results obtained in section 2, a global dry spectral primitive equation model is run and its output is analyzed. In section 3 , the model and model experiments are discussed and the numerical solutions are compared to the solutions obtained in section 2. In section 4, the model response in the presence of a simple frictional boundary layer is investigated. The discussion and conclusions are presented in section 5 .

\section{Basic equations and the solutions}

The most convenient (albeit idealized) parameterizations for dissipation are Rayleigh friction and Newtonian cooling. Under these parameterizations, the linearized, steady state equations for the tropical atmosphere are

$$
\begin{aligned}
\alpha u^{*}-\beta y v^{*} & =-\frac{\partial \Phi^{*}}{\partial x}, \\
\alpha v^{*}+\beta y u^{*} & =-\frac{\partial \Phi^{*}}{\partial y}, \\
\frac{\partial u^{*}}{\partial x}+\frac{\partial v^{*}}{\partial y}+\frac{1}{\rho_{0}} \frac{\partial\left(\rho_{0} w^{*}\right)}{\partial z^{*}} & =0 \\
\gamma \frac{\partial \Phi^{*}}{\partial z^{*}}+N^{2} w^{*} & =\frac{\kappa J}{H},
\end{aligned}
$$

where $u^{*}\left(v^{*}\right)$ is the eastward (northward) velocity; $w^{*}$ the vertical velocity in log pressure coordinates; $\Phi^{*}$ the geopotential; $\rho_{0}$ the basic density; $\kappa$ the gas constant $R$ divided by the heat capacity $c_{p} ; J$ the external heating rate per unit mass per unit time; $N^{2}$ the buoyancy frequency squared; $H$ the scale height; $\beta=2 \Omega / a$, where $\Omega$ and $a$ are the earth's rotation rate and radius, respectively; $\alpha$ is the Rayleigh friction rate; and $\gamma$ is the Newtonian cooling rate.

Equations (2.1) use an equatorial beta-plane approximation to the spherical earth and are standard for studying the tropical atmosphere (see, e.g., Holton 1992). While some studies (e.g., Miller and Lindzen 1992) showed that the strong (local) mean flow may contribute substantially to the organization of tropical rainfall in the tropical Africa (the local response), others (e.g., Robertson and Frankignoul 1990) showed that the moderate zonal mean easterlies [of a few meters per second as observed; see Fig. 5.10 of Lindzen (1990)] in the tropical troposphere do not affect significantly the planetary-scale response to the deep heating. Since the deep heating mainly project onto those vertical modes that have a zonal propagation speed of a few tens of meters per second (Wu et al. 2000), we expect that the moderate zonal mean easterlies do not have significant impact on the forced response. Therefore, to simplify the algebra, we assume a motionless basic state and an isothermal atmosphere of temperature $T_{s}$ although the generalization to a nonisothermal atmosphere is straightforward. Hence, the basic density $\rho_{0}$ satisfies

$$
\rho_{0}\left(z^{*}\right)=\rho_{0}(0) e^{-z^{*} / H} .
$$

To make our task easier we set both the Rayleigh friction rate $\alpha$ and the Newtonian cooling rate $\gamma$ as constants as in other studies (e.g., Geisler 1981).

To simplify Eqs. (2.1), we introduce the following mass-weighted variables, $u=\rho_{0}^{1 / 2} u^{*}, v=\rho_{0}^{1 / 2} v^{*}, w=$ $\rho_{0}^{1 / 2} w^{*}$, and $\Phi=\rho_{0}^{1 / 2} \Phi^{*}$, and substitute them into Eqs. (2.1) using Eq. (2.2) to obtain

$$
\begin{aligned}
\alpha u-\beta y v & =-\frac{\partial \Phi}{\partial x}, \\
\alpha v+\beta y u & =-\frac{\partial \Phi}{\partial y}, \\
\frac{\partial u}{\partial x}+\frac{\partial v}{\partial y}+\left(\frac{\partial}{\partial z}-\frac{1}{2 H}\right) w & =0 \\
\gamma\left(\frac{\partial}{\partial z}+\frac{1}{2 H}\right) \Phi+w N^{2} & =Q
\end{aligned}
$$

where $Q=\rho_{0}^{1 / 2} \kappa J / H$.

Equations (2.3a)-(2.3d) are combined to form a single equation for meridional velocity:

$$
\begin{aligned}
& \frac{\gamma}{N^{2} \alpha}\left(\frac{\partial^{2}}{\partial z^{2}}-\frac{1}{4 H^{2}}\right)\left(\beta^{2} y^{2}+\alpha^{2}\right) v+\left[\left(\frac{\partial^{2}}{\partial x^{2}}+\frac{\partial^{2}}{\partial y^{2}}\right)+\frac{\beta}{\alpha} \frac{\partial}{\partial x}\right] v \\
& =\left(\beta y \frac{\partial}{\partial x}-\alpha \frac{\partial}{\partial y}\right) \frac{1}{N^{2} \alpha}\left(\frac{\partial}{\partial z}-\frac{1}{2 H}\right) Q
\end{aligned}
$$

The presence of Rayleigh friction in Eq. (2.4) makes it impossible to find a complete set of meridional eigenfunctions that satisfy the boundary conditions at infinity, assuming also a Fourier representation in the zonal direction. Hence, it is difficult to use a modal approach to derive a nonhomogeneous ordinary differential equation and then to obtain the Green's functions response in the vertical. Alternative approaches must be used to reveal the properties of the solutions to Eqs. (2.3). One method is to find the solutions in different parameter regimes. To do that, we consider two sub- 


\section{Newtonian-Cooling-Alone-Case}
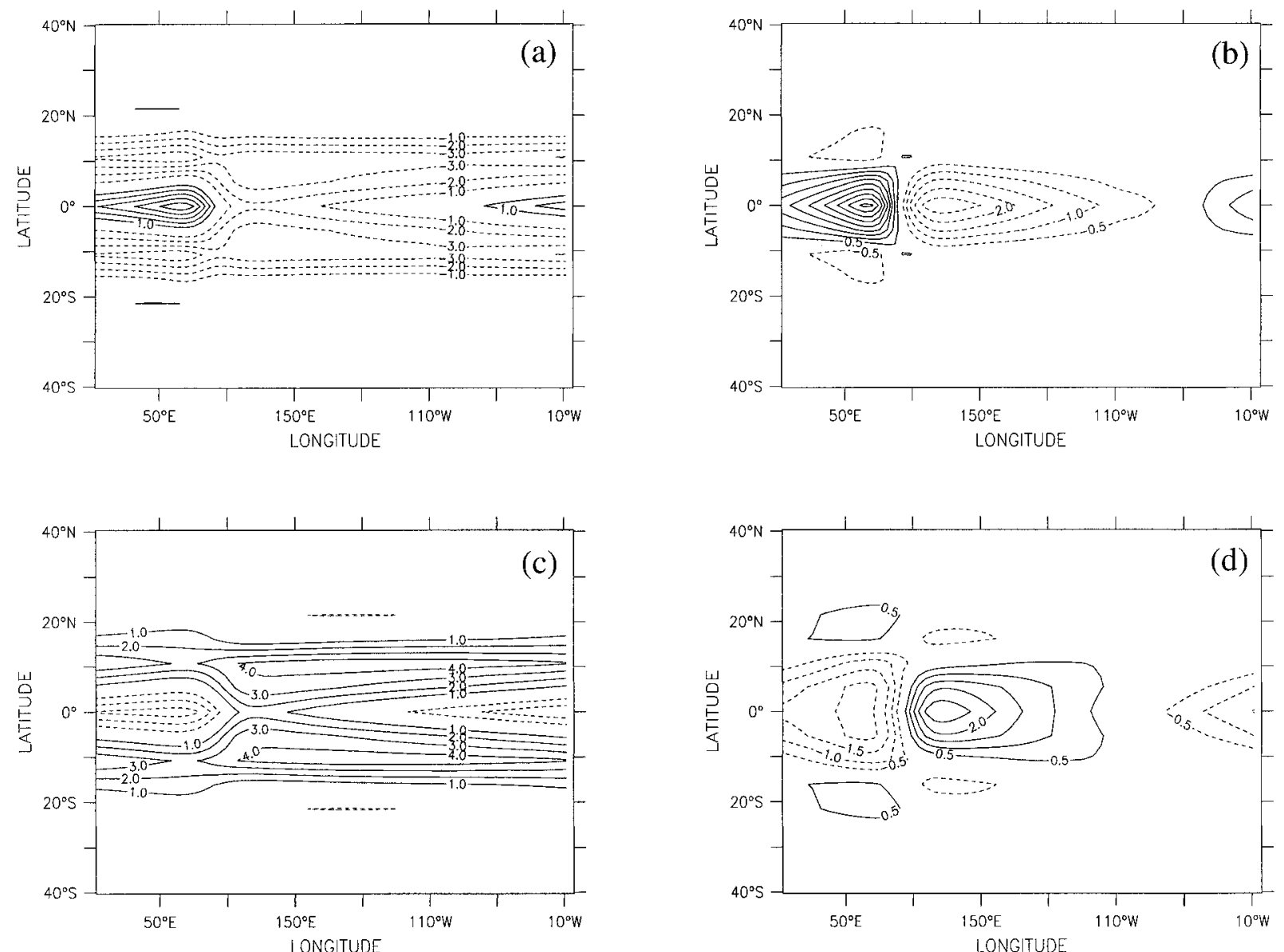

FIg. 6. Zonal velocities at different heights for the Newtonian cooling alone case. (a) and (c) Zonal velocities at 675 and $325 \mathrm{hPa}$, respectively. (b) and (d) The same as (a) and (c) but with the zonal mean removed. Contour interval is $1.0 \mathrm{~m} \mathrm{~s}^{-1}$ in (a) and (c) and $0.5 \mathrm{~m}$ $\mathrm{s}^{-1}$ in (b) and (d). The zero contour lines are not shown.

systems of Eqs. (2.3a)-(2.3d). In one subsystem, we assume that the thermal damping is not effective so that Rayleigh friction is the only mechanism able to dissipate energy (the Rayleigh friction alone case). In the second subsystem, only Newtonian cooling is assumed to dissipate energy (the Newtonian cooling alone case). The equations for these two systems are

$$
\begin{aligned}
\alpha u-\beta y v & =-\frac{\partial \Phi}{\partial x}, \\
\alpha v+\beta y u & =-\frac{\partial \Phi}{\partial y}, \\
\frac{\partial u}{\partial x}+\frac{\partial v}{\partial y}+\left(\frac{\partial}{\partial z}-\frac{1}{2 H}\right) w & =0, \\
w N^{2} & =Q, \quad \text { and }
\end{aligned}
$$

$$
\begin{aligned}
-\beta y v & =-\frac{\partial \Phi}{\partial x}, \\
\beta y u & =-\frac{\partial \Phi}{\partial y}, \\
\frac{\partial u}{\partial x}+\frac{\partial v}{\partial y}+\left(\frac{\partial}{\partial z}-\frac{1}{2 H}\right) w & =0, \\
\gamma\left(\frac{\partial}{\partial z}+\frac{1}{2 H}\right) \Phi+w N^{2} & =Q .
\end{aligned}
$$

The boundary conditions are standard: in the zonal direction, zonal periodicity is assumed; in the meridional direction, the atmosphere is assumed to be undisturbed far away from the external heat source; and in the vertical, there is no vertical motion at the surface and the "radiation condition" is applied at infinity. 


\section{a. The Newtonian cooling alone case}

Combining Eqs. (2.6a)-(2.6d) leads to

$\frac{\gamma}{N^{2}} \beta y^{2}\left(\frac{\partial^{2}}{\partial z^{2}}-\frac{1}{4 H^{2}}\right) \Phi+\frac{\partial \Phi}{\partial x}=\frac{\beta y^{2}}{N^{2}}\left(\frac{\partial}{\partial z}-\frac{1}{2 H}\right) Q$.

Terms with scale height $H$ in Eq. (2.7) are very small for the response of the system under the forcing considered in this paper, and these terms can be omitted (these terms come from basic density variation in the vertical direction).

Lindzen (1968) first examined the role of Newtonian cooling on tides in the Tropics. In that paper, he found that when the Newtonian cooling is the only damping process, the assumption of zonal periodicity does not change the meridional eigenfunctions, so one can use the Fourier transform in the zonal direction. Hence, we expand $Q$ and $\Phi$ as follows:

$$
\begin{aligned}
Q & =\sum_{k} \sum_{n} Q_{n, k}(z) \phi_{n}(y) \exp (i k x) \quad \text { and } \\
\Phi & =\sum_{k} \sum_{n} \Phi_{n, k}(z) \phi_{n}(y) \exp (i k x),
\end{aligned}
$$

where

$$
\phi_{n}(y)=\exp \left(-\frac{y^{2}}{2 L^{2}}\right) H_{n}\left(\frac{y}{L}\right)
$$

$k$ and $n$ are nonnegative integers, and $L$ is a constant that is independent of $n$.

Although $\left\{\phi_{n}(y)\right\}$ is a complete set for any given $L$, in practice, the selection of $L$ in Eq. (2.10) is not arbitrary. For example, if $Q$ is Gaussian with a scale $L_{f}$ and we select $L=L_{f}$, then only the $n=0$ terms appear on the right-hand side of Eq. (2.8). If we select any other $L$, we will need many $\phi_{n}(y)$ to obtain accurate expansions. In this study, we identify $L$ with the characteristic meridional scale of the heat source, $L=L_{f}$.

The recursive relationship for the parabolic cylinder function $\phi_{n}(y)$ is

$$
y^{2} \phi_{n}=\frac{L^{2}}{4}\left[\phi_{n+2}+2(2 n+1) \phi_{n}+4 n(n-1) \phi_{n-2}\right] .
$$

Substituting Eqs. (2.8), (2.9), and (2.11) into Eq. (2.7), we obtain

$$
\begin{gathered}
\left(\frac{\partial^{2}}{\partial z^{2}}-\frac{1}{4 H^{2}}\right)\left[\Phi_{n-2, k}+2(2 n+1) \Phi_{n, k}+4(n+1)(n+2) \Phi_{n+2, k}\right]+\frac{4 N^{2}}{\beta \gamma L^{2}} i k \Phi_{n, k} \\
=\frac{1}{\gamma}\left(\frac{\partial}{\partial z}-\frac{1}{2 H}\right)\left[Q_{n-2, k}+2(2 n+1) Q_{n, k}+4(n+1)(n+2) Q_{n+2, k}\right] .
\end{gathered}
$$

Equation (2.12) is true for any nonnegative integer $n$. For planetary-scale motions, when $L_{f}$ is large, the term with $k$ is relatively small and Eq. (2.12) simplifies to

$$
\left(\frac{\partial}{\partial z}+\frac{1}{2 H}\right) \Phi_{n, k}=\frac{1}{\gamma} Q_{n, k}
$$

The heating term $Q$ represents the large-scale cumulus heating of the tropical atmosphere. Hence, $Q$ is zero below the lifting condensation level $Z_{l}$ and above the cloud top $Z_{t}$ (somewhere close to the tropopause). Thus we find

$$
\left(\frac{\partial}{\partial z}+\frac{1}{2 H}\right) \Phi_{n, k}=0
$$

both below the lifting condensation level $Z_{l}$ and above the cloud top $Z_{t}$. The solutions to Eq. (2.14) automatically satisfy the lower boundary condition since Eq. (2.14) has the same form as the lower boundary condition. Combining Eqs. (2.6b), (2.9), and (2.14) leads to

$$
\left(\frac{\partial}{\partial z}+\frac{1}{2 H}\right) u=0, \text { for } z<Z_{l} \text { or } z>Z_{t} .
$$

Note that $u$ is the mass-weighted eastward (zonal) velocity. For eastward (zonal) velocity $u^{*}$ itself, Eq. (2.15a) simplifies to

$$
\frac{\partial u^{*}}{\partial z}=0, \text { for } z<Z_{l} \text { or } z>Z_{t} \text {. }
$$

Equation (2.15b) implies that from the cloud base to the surface where the external forcing is zero, the zonal velocity is vertically uniform. Since the zonal velocity is generally not zero at the cloud base, this result implies that surface wind can be affected by elevated thermal forcing when thermal damping dominates. If the zonal velocity at the cloud top is not zero, we would also expect the almost vertically uniform zonal velocity above the cloud top.

Physically, Newtonian cooling parameterizes the processes that drive the perturbed atmosphere to its thermal equilibrium state. Since temperature is proportional to $\partial \Phi^{*} / \partial z$ in an isothermal atmosphere (this relation is also 


\section{Newtonian-Cooling-Alone-Case}
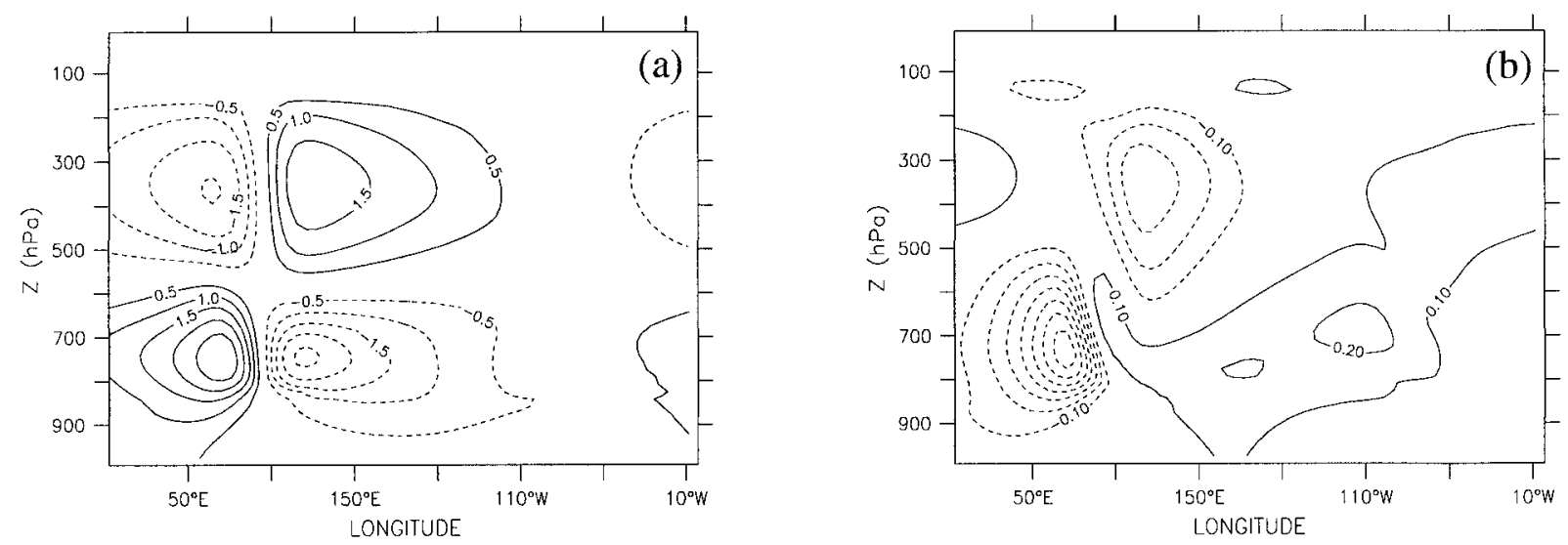

FIG. 7. Zonal velocity anomalies (i.e., the zonal wind minus the zonal mean zonal wind) at different latitude bands for the Newtonian cooling alone case. (a) The latitude band from the equator to $10^{\circ} \mathrm{N}$, and (b) the latitude band from $10^{\circ}$ to $20^{\circ} \mathrm{N}$. Contour interval is $0.5 \mathrm{~m}$ $\mathrm{s}^{-1}$ in (a) and $0.1 \mathrm{~m} \mathrm{~s}^{-1}$ in (b). The zero contour lines are not shown.

a very accurate approximation in the case of a nonisothermal atmosphere), Newtonian cooling relaxes $\partial \Phi^{*} / \partial z$ to zero in the layers where the external heating rate is zero. Hence, we can expect that the vertical derivative of horizontal velocity to be zero in nonheated layers where the Newtonian cooling dominates. This also indicates that Newtonian cooling in a three-dimensional atmosphere acts differently from the way it does in a two-dimensional shallow water system where Newtonian damping mainly relaxes the amplitude of the perturbation of geopotential forced by external heating.

\section{b. The Rayleigh friction alone case}

The governing equations for this case are Eqs. (2.5a)(2.5d). If the heating $Q(x, y, z)$ can be expressed as the product of horizontal $Q(x, y)$ and vertical $S(z)$ structures for which the first-order vertical derivatives at $Z_{l}$ and $Z_{t}$ exist (so that the vertical boundary conditions can be satisfied), we can write all the variables as $u=$ $\underset{\sim}{u}(x, y)(\partial / \partial z-1 / 2 H) S, v=\underset{\sim}{v}(x, y)(\partial / \partial z-1 / 2 H) S, \Phi$ $\stackrel{\sim}{=} \Phi(x, y)(\partial / \partial z-1 / 2 H) S$, and $w=\underset{\sim}{w S}$. Then, Eqs. (2.5a)-(2.5d) become

$$
\begin{aligned}
& \alpha \underset{\sim}{u}-\beta y \underset{\sim}{v}=-\frac{\partial \underset{\sim}{\Phi}}{\partial x},
\end{aligned}
$$

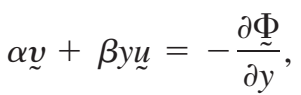

$$
\begin{aligned}
& \frac{\partial \underset{\sim}{u}}{\partial x}+\frac{\partial \underset{\sim}{v}}{\partial y}=-\frac{\underset{\sim}{Q}}{N^{2}}, \\
& \underset{\sim}{w} N^{2}=\underset{\sim}{Q} .
\end{aligned}
$$

Equations (2.16a)-(2.16c) are a two-dimensional system that is easily solved analytically. Since the response is proportional to $S(z)$, it is limited to the layers where the heating is not zero. Hence, in this model an elevated large-scale heat source cannot affect the surface circulation in the Tropics when Rayleigh friction dominates. Combining Eqs. (2.16a)-(2.16c) we obtain equations of $\underset{\sim}{u}$ and $\underset{\sim}{v}$ in terms of $Q$ :

$\left[\left(\frac{\partial^{2}}{\partial x^{2}}+\frac{2 \beta}{\alpha} \frac{\partial}{\partial x}\right)+\frac{\partial^{2}}{\partial y^{2}}\right] \underset{\sim}{u}=\frac{1}{N^{2}}\left(\frac{\partial}{\partial x}+\frac{\beta y}{\alpha} \frac{\partial}{\partial y}+\frac{2 \beta}{\alpha}\right) \underset{\sim}{\underset{\sim}{Q}, \quad \text { and }}$

$\left[\left(\frac{\partial^{2}}{\partial x^{2}}+\frac{\beta}{\alpha} \frac{\partial}{\partial x}\right)+\frac{\partial^{2}}{\partial y^{2}}\right] \underset{\sim}{v}=\frac{1}{N^{2}}\left(\frac{\partial}{\partial y}-\frac{\beta y}{\alpha} \frac{\partial}{\partial x}\right) \underset{\sim}{\underset{\sim}{(}}$

To solve Eq. (2.17a) or Eq. (2.17b), we use a Fourier transform in the zonal direction:

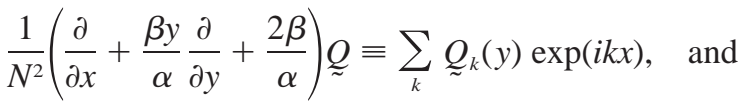

$$
\underset{\sim}{u} \equiv \sum_{k} \underset{\sim}{u_{k}}(y) \exp (i k x)
$$

Substituting Eqs. (2.18a) and (2.18b) into Eq. (2.17a), we obtain a linear ordinary differential equation for $\underset{\sim}{\underset{\sim}{u} \text { : }}$ :

$$
\left[\frac{d^{2}}{d y^{2}}-\left(k^{2}-2 \beta \frac{i k}{\alpha}\right)\right] \underset{\sim}{\underset{u}{k}}(y)=\underset{\sim}{Q_{k}(y)} .
$$

The character of the solution to Eq. (2.19) is determined by the real part of the square root of $k^{2}-2 \beta i k / \alpha$, which is related to the meridional decay scale of the 
forced signal. For the large-scale motions being considered in this paper, $\beta / \alpha$ is often larger than the wavenumber $k$. Hence, enhancing the momentum damping rate $(\alpha)$ increases the distance the forced signals can propagate poleward from the heat source. When $\alpha$ is small, the forced signals do not propagate far away from the heat source, and the response is meridionally confined to a zone near the heat source.

\section{c. Discussion}

The overall vertical response to an elevated heat source in the presence of both Rayleigh friction and Newtonian cooling is expected to be an amalgamation of these two extreme cases and will depend on the rates of both Rayleigh friction and Newtonian cooling (see also section 4). The observational studies by Reed and Recker (1971) and Wallace (1971) suggests that the thermal damping term is at least an order of magnitude smaller than the large-scale cumulus heating term. Hence, from the discussion above, we expect that the vertical response to an elevated large-scale heat source most resembles that of the Rayleigh friction alone case. It also implies that under large enough mechanical damping, we may approximate the linear, vertically semibounded system [(2.3a)-(2.3d)] by applying an artificial lid far above $Z_{t}$ and then use vertical normal modes to expand the variables and heating to obtain the approximate solutions.

In general, the timescale of Newtonian cooling is of the order of 2 weeks and that of Rayleigh friction is about 5-10 days. When the damping terms are assumed to represent cumulus friction and vertical transport of heat by cumulus convection, both the effective Rayleigh friction and Newtonian cooling can be of the order of a day or less in the Tropics. Holton and Colton (1972) point out that the damping used in a linear model needs to be much stronger (less than a day) than people generally believe to include the effect of the nonlinearity in the tropical atmosphere.

\section{Numerical experiments}

The analytical results in section 2 demonstrate the different roles of Rayleigh friction and Newtonian cooling in response to steady tropical heating. In this section, we analyze model output from two idealized numerical experiments, in which the prescribed heating is identical. The first experiment is called the the Newtonian cooling alone case, in which the model has only Newtonian cooling as the dissipation mechanism. In the second experiment, Rayleigh friction replaces Newtonian cooling (the Rayleigh friction alone case). We will examine the circulation patterns associated with both cases and determine to what extent the model results are consistent with the qualitative analysis in section 2 .

\section{a. The model}

The model we use is the dry version of the primitive equation model developed by Saravanan and McWilliams (1995). The model assumes that the planetary surface has no topography, and the solutions are calculated using spectral methods and a pressure coordinate. The divergent shallow water mode with equivalent barotropic vertical structure is excluded by imposing a rigid lid at the top of the model atmosphere. The model results show that the solutions are almost independent of the artificial lid, as long as the lid is well above the top of the isolated heat source.

Our interests are on the impact of an elevated largescale heat source on the tropical circulations, especially on the surface winds. We do not consider the potential for feedback between large-scale motion and the cumulus convection, nor do we explicitly simulate smalland mesoscale features of cumulus convection. Furthermore, to facilitate the interpretation of the results, we will take the resting atmosphere to be $300 \mathrm{~K}$ isothermal and exclude an explicit boundary layer. The upper and lower boundary conditions are $w^{*}=0$. This boundary condition is consistent with Eq. (2.5d) for the Rayleigh friction alone case and Eqs. (2.6d) and (2.14) for the Newtonian cooling alone case.

The heat source is prescribed to be

$$
Q=\exp \left[-\left(\lambda-\lambda_{0}\right)^{2} / \lambda_{L}^{2}\right] \exp \left(-\phi^{2} / \phi_{L}^{2}\right) F(p),
$$

where $\lambda$ is longitude, $\lambda_{0}$ is reference longitude with value of $93^{\circ} \mathrm{E}, \lambda_{L}=20^{\circ}, \phi$ is latitude, and $\phi_{L}=11^{\circ}$. For simplicity, $F(p)$ is defined as

$$
F(p)= \begin{cases}0, & p \geq p_{b}, \\ F_{0} \sin \left(\frac{p-p_{t}}{p_{b}-p_{t}} \pi\right), & p_{t}<p<p_{b}, \\ 0, & p \leq p_{t},\end{cases}
$$

where $p$ is pressure, $p_{t}$ is the pressure at the top of the forcing, and $p_{b}$ is the pressure at the bottom of the forcing. We assume that cloud top is at $160 \mathrm{hPa}$ and cloud base is at $840 \mathrm{hPa}$. To examine the vertical structure of the low-level flows clearly, the cloud base taken in this paper is considerably higher than observed (Mapes and Houze 1995). Further, $F_{0}$ is the maximum heating rate so that $F$ corresponds to $10 \mathrm{~mm}$ of precipitation per day. The shape of $Q$ is displayed in Fig. 1, along with the locations of the cross sections (labeled $W, C$, $E, P_{i}$, and $H_{i}$ ) that will be used to examine the results. Figure 1a is the horizontal structure (in which only part of the horizontal domain is shown) and Fig. $1 \mathrm{~b}$ is the vertical distribution of the heating.

The vertical resolution is set to 60 layers of the equal thickness (in pressure). The horizontal resolution is T21, which corresponds to 64 grid points zonally and 33 grid points meridionally. 


\section{Newtonian-Cooling-Alone-Case}
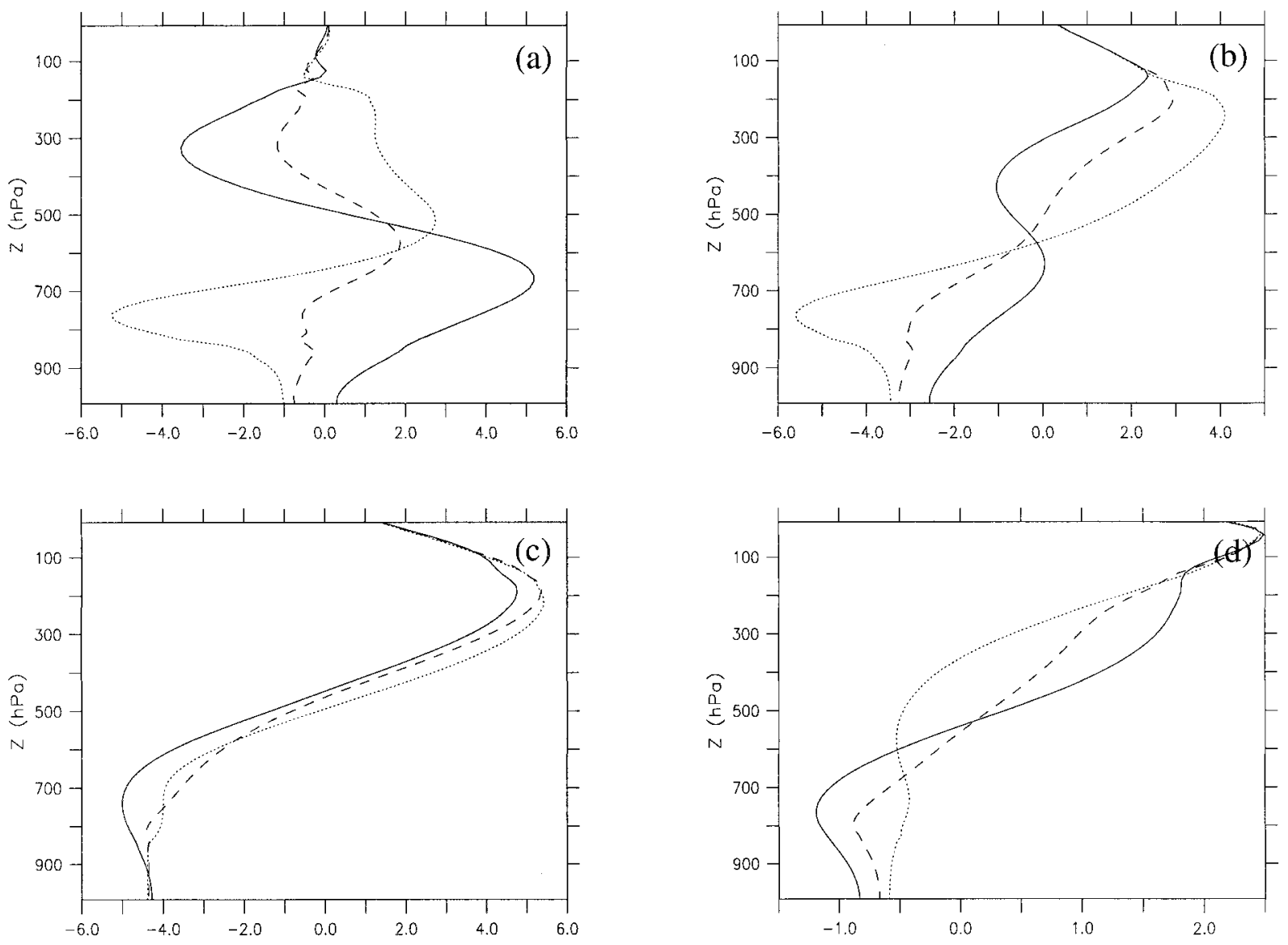

FIG. 8. Vertical profiles of zonal velocity at different locations for the Newtonian cooling alone case. (a) At the equator, (b) at $5.4^{\circ} \mathrm{N},(\mathrm{c})$ at $11.7^{\circ} \mathrm{N}$, (d) at $16.1^{\circ} \mathrm{N}$. Solid lines are at W; dashed lines are at C; dotted lines are at E. The unit along the abscissa is $1.0 \mathrm{~m} \mathrm{~s}^{-1}$.

\section{b. Experiments}

Two model runs are performed. The first run has Rayleigh friction only: no other damping is applied to the model. The Rayleigh friction rate is taken to be $\alpha^{-1}=$ 10 days. The second run examines the opposite end of the parameter regime, where the momentum damping is set to zero and Newtonian cooling is the only damping in the model. Generally, there are two approaches to determine the value of Newtonian cooling rate. The first approach is based on the physical processes, such as radiative cooling. In this approach, the Newtonian cooling rate is estimated to be about 2 weeks or longer. Because the Newtonian cooling must also account for additional thermal dissipative processes (e.g., turbulence, convection), an appropriate (effective) Newtonian cooling in a primitive equation model may be much greater than the inverse of the radiative time scale. Hence, we determine the Newtonian cooling rate as follows. In the Tropics, the maximum temperature perturbation observed on seasonal timescale is less than $2^{\circ} \mathrm{C}$
(Bantzer and Wallace 1996). To keep the model temperature perturbation comparable to the observations, the Newtonian cooling rate is taken to be $\gamma^{-1}=2.5$ days, a value comparable to that used in other models (e.g., Gill 1980; Geisler 1981). The model results discussed are not qualitatively dependent on the value of Newtonian cooling rate in the Newtonian cooling alone case.

We run both model experiments for 400 model days. Figure 2 plots the global kinetic energy and the global perturbed potential energy for both cases. Since there is no thermal damping in the Rayleigh friction alone case, the global potential energy grows linearly while the global kinetic energy approaches a steady state. A physical scenario corresponding to this case is that the thickness between two isobaric planes increases as it is heated while the slope of the two planes remains unchanged. For the Newtonian cooling alone case, the global potential energy goes rapidly to a steady state, while the global kinetic energy equilibrates at a much 


\section{Newtonian-Cooling-Alone-Case}
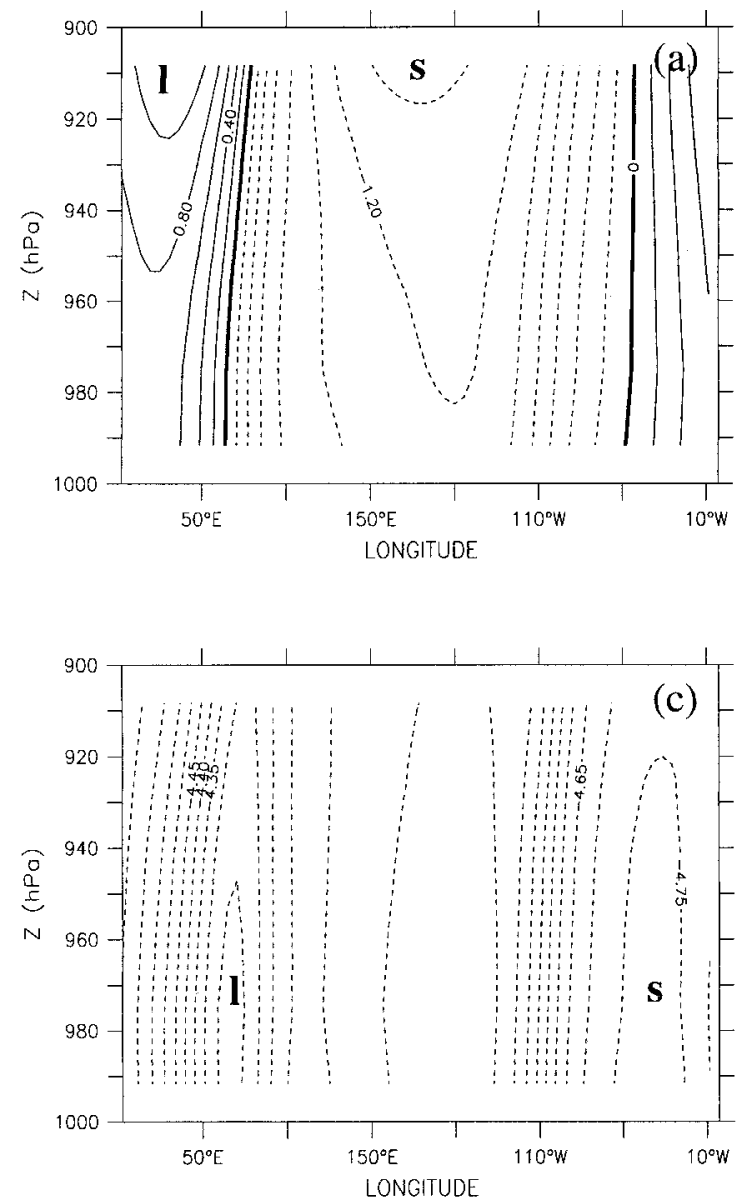
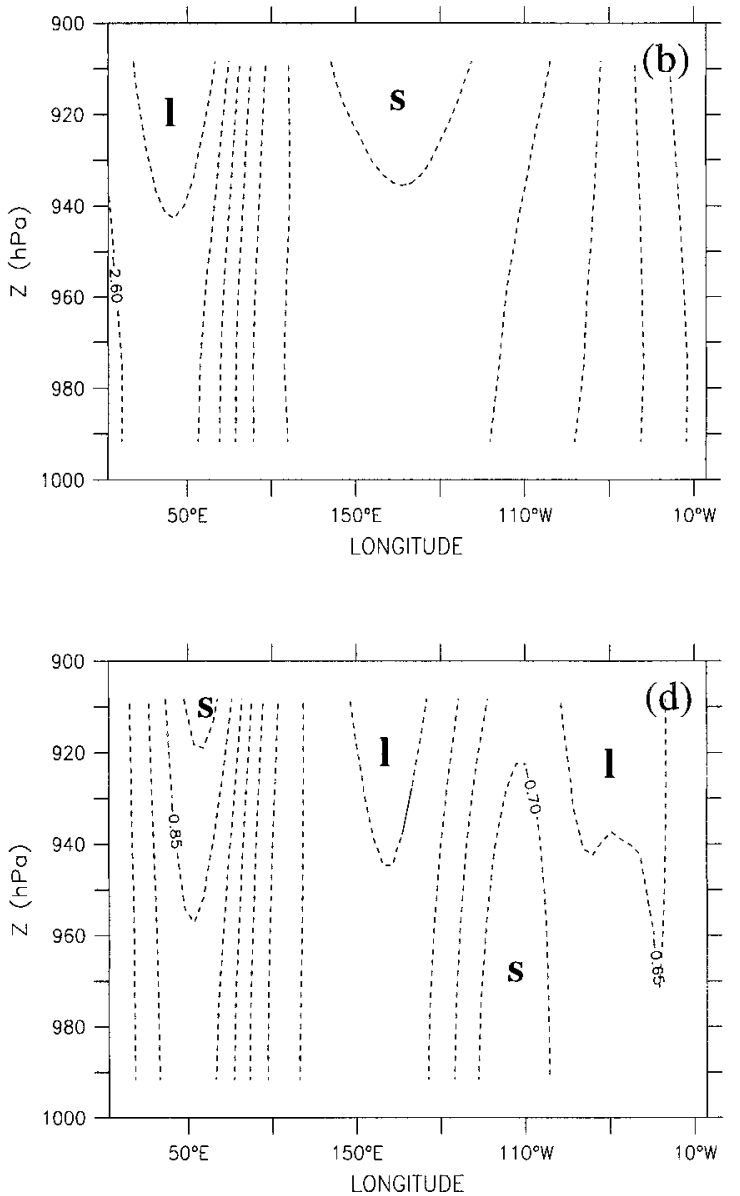

FIG. 9. Zonal velocities at different latitudes below $900 \mathrm{hPa}$ for the Newtonian cooling alone case. (a) At the equator, (b) at $5^{\circ} \mathrm{N}$; (c) $11^{\circ} \mathrm{N}$, (d) at $16^{\circ} \mathrm{N}$. In each panel, "l" represents a maximum westerly (or minimum easterly) center, and "s" represents a maximum easterly (or minimum westerly) center. Contour intervals are $0.2 \mathrm{~m} \mathrm{~s}^{-1}$ in (a) and (b), and $0.05 \mathrm{~m} \mathrm{~s}^{-1}$ in (c) and (d).

slower rate. After about 100 model days only the magnitude of horizontal velocity is increasing in the Newtonian cooling alone case: the three-dimensional structure of horizontal velocity is robust for integrations larger than 100 model days.

\section{c. Analysis of model results}

\section{1) The Newtonian cooling Alone CASE}

Figure 3 shows the zonal velocity at different longitudes. Although the zonal winds in the upper troposphere are crudely opposite to that in the lower troposphere at almost every latitude, there are some differences between the vertical structure of the response to the east and the west of the heat source (see also Fig. 4). To the west of the heat source (Fig. 3a), the level of zero wind is located at about $450 \mathrm{hPa}$. In the lower troposphere, a narrow but strong westerly band sits be- tween two strong easterly bands to the north and the south, and the maximum width of this westerly band is about $10^{\circ}$ in latitude. The upper troposphere is dominated by westerlies, although there is a band of relatively weak easterly wind just above the westerly jet in the lower half of the troposphere in the equatorial zone. To the east of the heat source (Fig. 3c), the lower troposphere is dominated by easterlies and the upper troposphere by westerlies, and the zero wind level is much lower than it is to the west of the heat source. At the center of the heating region (Fig. 3b), the zonal winds in the upper troposphere resembles those to the west of the heating, while the zonal winds in the lower troposphere resembles those to the east of the heating. At every longitude, the response is meridionally confined to where the heating takes place: poleward of $20^{\circ}$ latitude, the signal is vanishingly small. This result is consistent with Eq. (2.13).

At the surface, easterlies are predominant. The mag- 


\section{Rayleigh-Friction-Alone-Case}
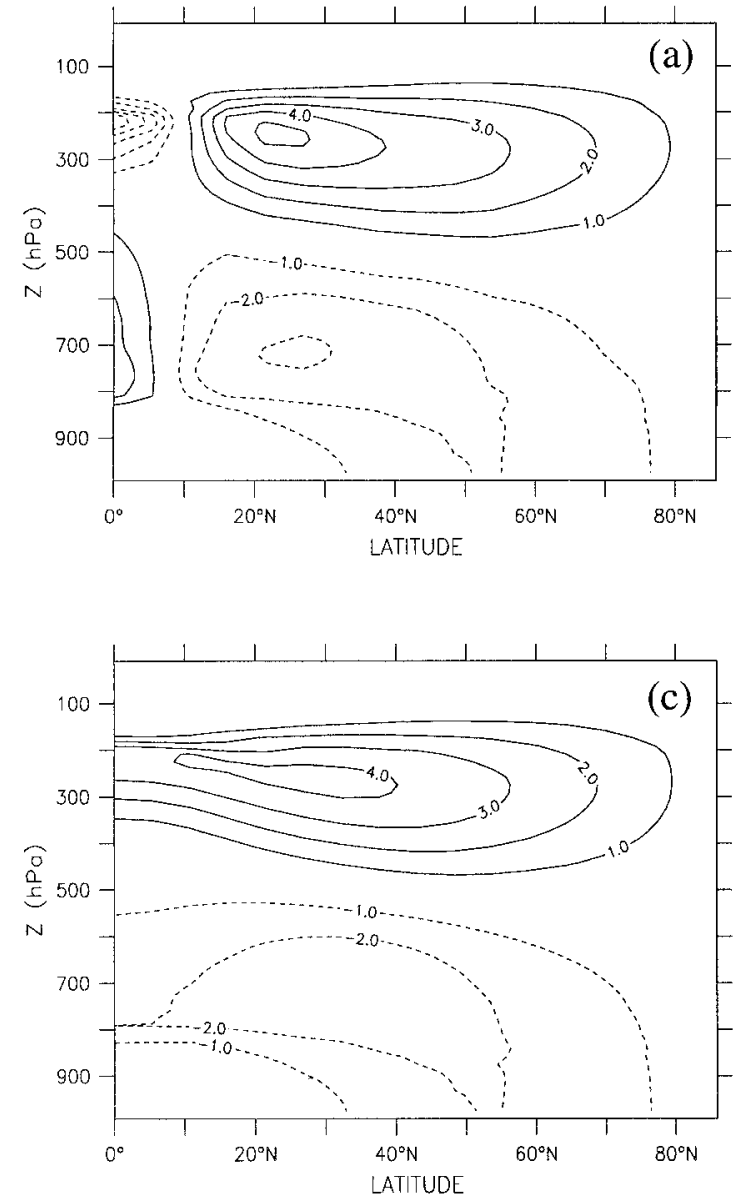

nitude of easterlies increases with increasing latitude from the equator to about $10^{\circ}$ where the easterlies reach their maximum. Poleward of $10^{\circ}$ latitude, the magnitude of easterlies decreases dramatically. To the west of the heating region, the surface winds are very weak in a very narrow band (about $2^{\circ}$ ) centered about the equator.

The winds at the top of the model atmosphere are not zero. However, the response to such an isolated heat source is not sensitive to where the lid is imposed as long as it is imposed higher than $100 \mathrm{hPa}$ because the density above $100 \mathrm{hPa}$ is very small. Thus, there is little vertical energy propagation near the lid due to small wave density; hence, the total energy reflected by the artificial lid is negligible.

Figures 4 and 5 show the forced circulations and the temperature perturbations at different latitudes. Easterlies dominate in the lower troposphere; above, westerlies dominate almost everywhere except in a narrow meridional band along the equator. Large vertical velocity is significant equatorward of $10^{\circ}$ latitude, where heating is mainly balanced by adiabatic cooling due to

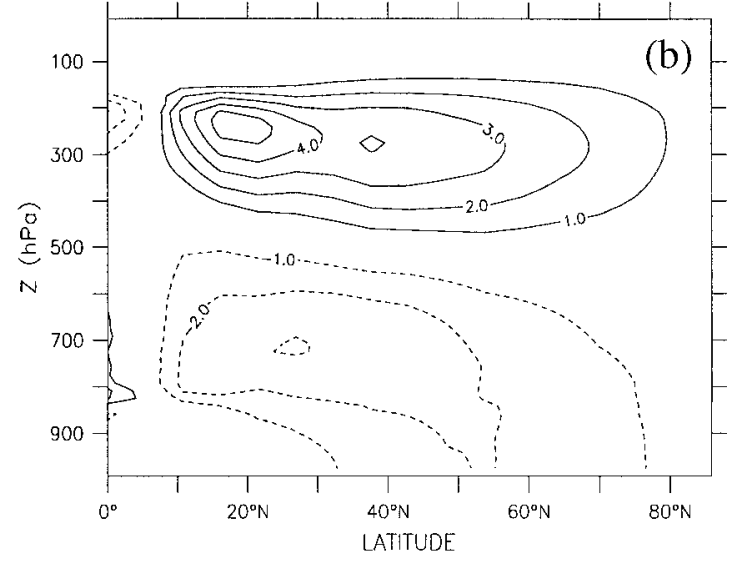

Fig. 10. Zonal velocities at different longitudes for the Rayleigh friction alone case. Solid lines represent westerlies, and dashed lines represents easterlies. (a) At $53.4^{\circ} \mathrm{E}$, (b) at $92.8^{\circ} \mathrm{E}$, (c) at $132.2^{\circ} \mathrm{E}$. Contour interval is $1.0 \mathrm{~m} \mathrm{~s}^{-1}$ in every panel. Zero contour lines are not shown.

upward motions. To the east of the forcing region, the meridional velocity is almost zero everywhere, while to the west the meridional velocity decreases very rapidly away from the heat source. Figure 5 shows the temperature perturbations at different latitudes. The temperature perturbations reach their maximum at the level of maximum heating. The isotherms are almost flat in the zonal direction at all the latitudes, especially in the upper troposphere, despite the large Newtonian cooling rate. The zonal overturning can be identified in Figs. $4 a, b$ but the two-cell structure is somewhat ill-defined along the equator.

The decoupling between the temperature and the horizontal wind (which does show decay away from the heating in the equatorial region) is quite clear in the previous figures. In Figs. 6b,d, the anomaly zonal winds do decay away from the heat source in the zonal direction, but the decay rate to the west of the heat source is similar to that to the east of the heat source, rather than being threefold as in Gill's solution. Finally, by removing the zonal mean from the zonal velocities then 


\section{Rayleigh-Friction-Alone-Case}
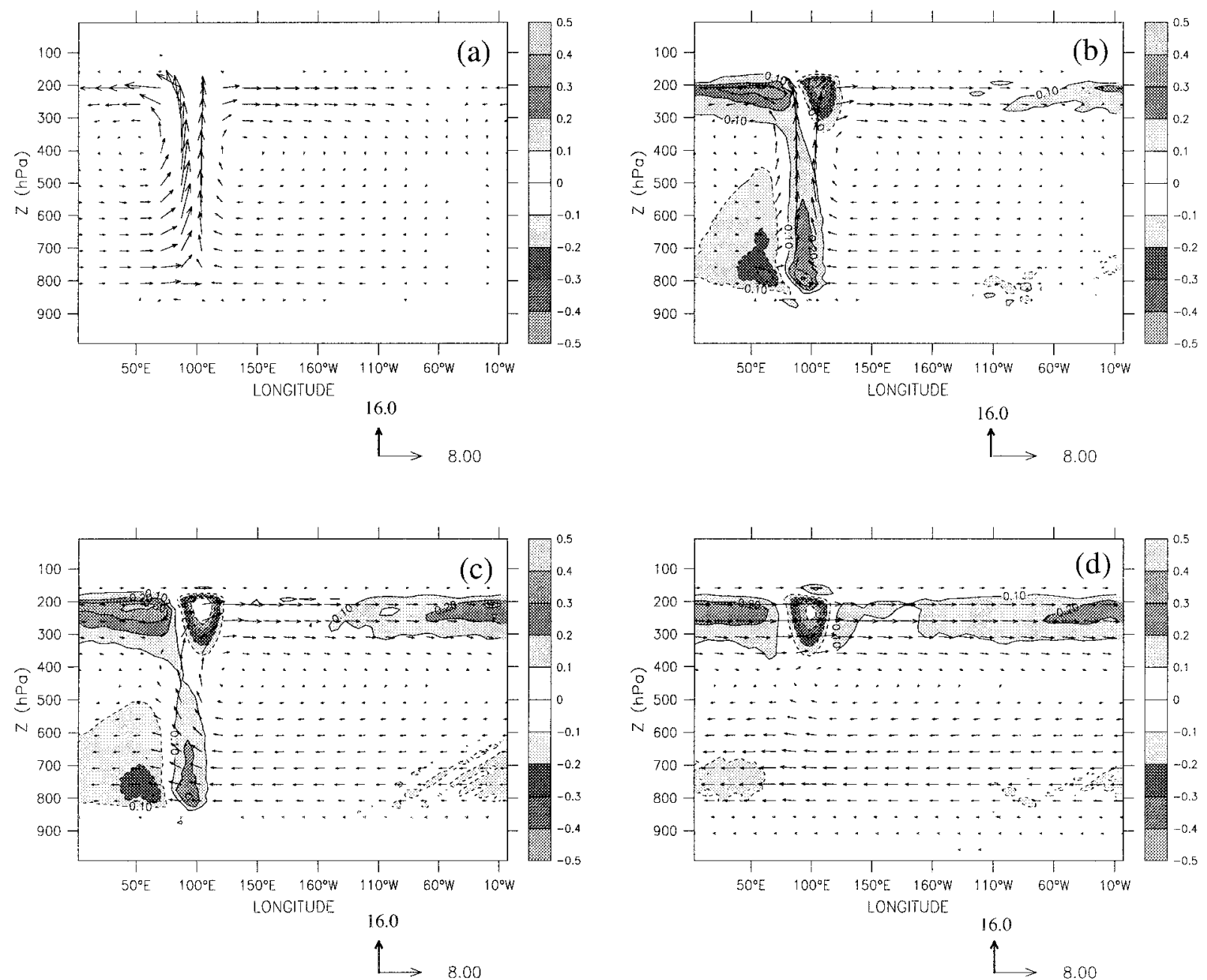

FIG. 11. The zonal overturning circulations at different latitudes for the Rayleigh friction alone case. The vector field of zonal velocity (with unit $\mathrm{m} \mathrm{s}^{-1}$ ) and vertical velocity $\left(-d p / d t\right.$ with unit $\mathrm{hPa}$ day ${ }^{-1}$ ) are superposed on the contoured meridional velocity (with contour interval $0.1 \mathrm{~m} \mathrm{~s}^{-1}$ ). The zonal velocity and the vertical velocity are scaled separately and are shown under each panel. (a) At the equator, (b) at $5.4^{\circ} \mathrm{N}$; (c) at $11.7^{\circ} \mathrm{N}$, (d) at $16.1^{\circ} \mathrm{N}$.

averaging over two $10^{\circ}$ latitude bands, one finds that the zonally overturning cells are confined to a narrow band along the equator (Fig. 7).

Vertical profiles of zonal velocity at different locations are shown in Fig. 8. It is clear that the vertical structure of zonal velocity varies dramatically at different locations. At about $10^{\circ} \mathrm{N}$, the low-level zonal velocity is almost as large as the zonal velocity at 750 $\mathrm{hPa}$. The zonal velocity at that latitude is also fairly uniform zonally. Another interesting feature is that zonal velocity is quasi-uniform with height below the bottom of the heat source, also evident in Fig. 9: below 900 $\mathrm{hPa}$, the zonal velocity is everywhere nearly uniform vertically. This generally agrees with the analytical result [Eq. (2.15)].

\section{2) The Rayleigh friction alone case}

As we anticipated from the analysis in section 2, for the Rayleigh friction alone case the atmospheric motion is confined in the vertical to where the heating takes place: below and above the heat source, the atmosphere is not affected by the heating. Figure 10 is the counterpart of Fig. 3 for the Rayleigh friction alone case. Compared to the narrow meridional response in the Newtonian cooling alone case (Fig. 3), we see that the response to the heating is global. Westerly jets are found in the subtropical upper troposphere to the west of the heat source. To the east of the heating, these two westerly jets merge to form a broad westerly flow extending from pole to pole in the upper troposphere. The flow in the lower tropo- 


\section{Rayleigh-Friction-Alone-Case}
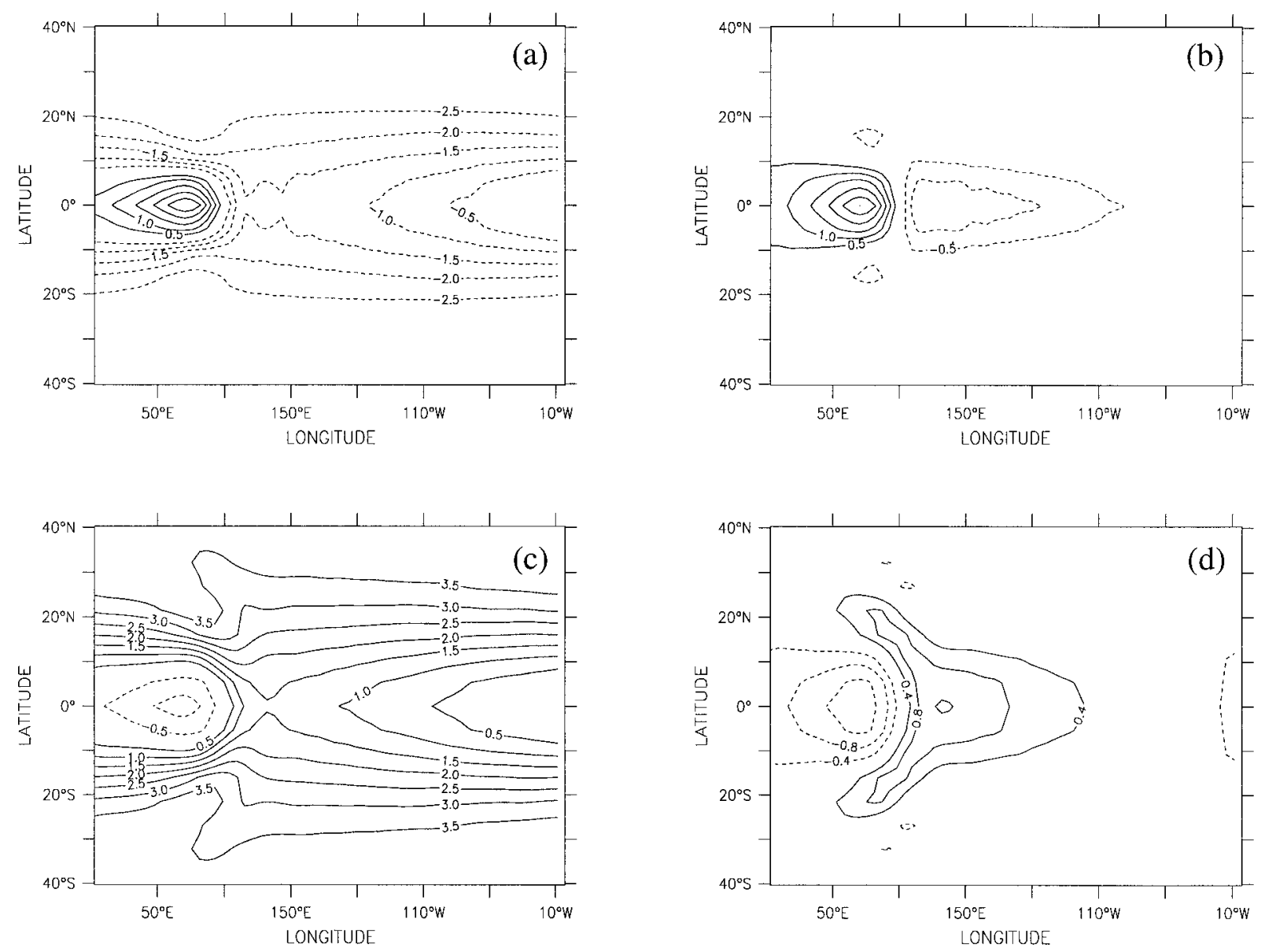

Fig. 12. Zonal velocities at different altitudes for the Rayleigh friction alone case. (a) and (c) Zonal velocities at 675 and $325 \mathrm{hPa}$, respectively. (b) and (d) The same as (a) and (c), respectively, but with the zonal mean removed. Contour intervals are $0.5 \mathrm{~m} \mathrm{~s}^{-1}$ in (a), (b), and (c), and $0.4 \mathrm{~m} \mathrm{~s}^{-1}$ in (d). The zero contour lines are shown.

sphere is similar in pattern to that in the upper atmosphere, albeit weaker and with opposite sign. The zonal flow below and above the heat source in the tropical region is vanishingly small, consistent with the analysis applied on an equatorial beta plane in section 2 .

The circulations at different latitudes are displayed in Fig. 11. A zonal overturning circulation is evident along the equator, especially to the east of the heating region. At higher latitudes, easterlies are found in the lower troposphere and westerlies dominate in the upper troposphere. The zonal decay away from the heating region is much clearer than in the Newtonian cooling alone case. The pattern of meridional velocity in the Rayleigh friction alone case is quite different from that in the Newtonian cooling alone case (cf. Fig. 4). Off the equator, the westward decay of the meridional velocity is more gradual than in the Newtonian cooling alone case: for example, at about $15^{\circ}$ latitude (Fig. 11d), we see poleward motion in the upper troposphere and an equatorward motion in the lower troposphere at almost every longitude except where the heat source is located.

As expected, the upward motion takes the shape of the heating in the equatorial region, while the downward motion is broad but very weak (Fig. 11a). Although the forcing is localized, isotherms are again flat in the zonal direction throughout the Tropics (not shown). Because there is no thermal damping, the amplitude of temperature perturbation is very large.

Figure 12 shows the zonal velocity at different heights. After its zonal mean is removed, we see that zonal wind is qualitatively similar to that described by Gill (1980). The zonally averaged quantities are shown in Fig. 13, which clearly illustrates a global Hadley circulation (cf. Fig 13a for the Newtonian cooling alone case). The flow below the heating is weak everywhere, evident from Figs. 11 and 13. 


\section{Rayleigh-Friction-Alone-Case}
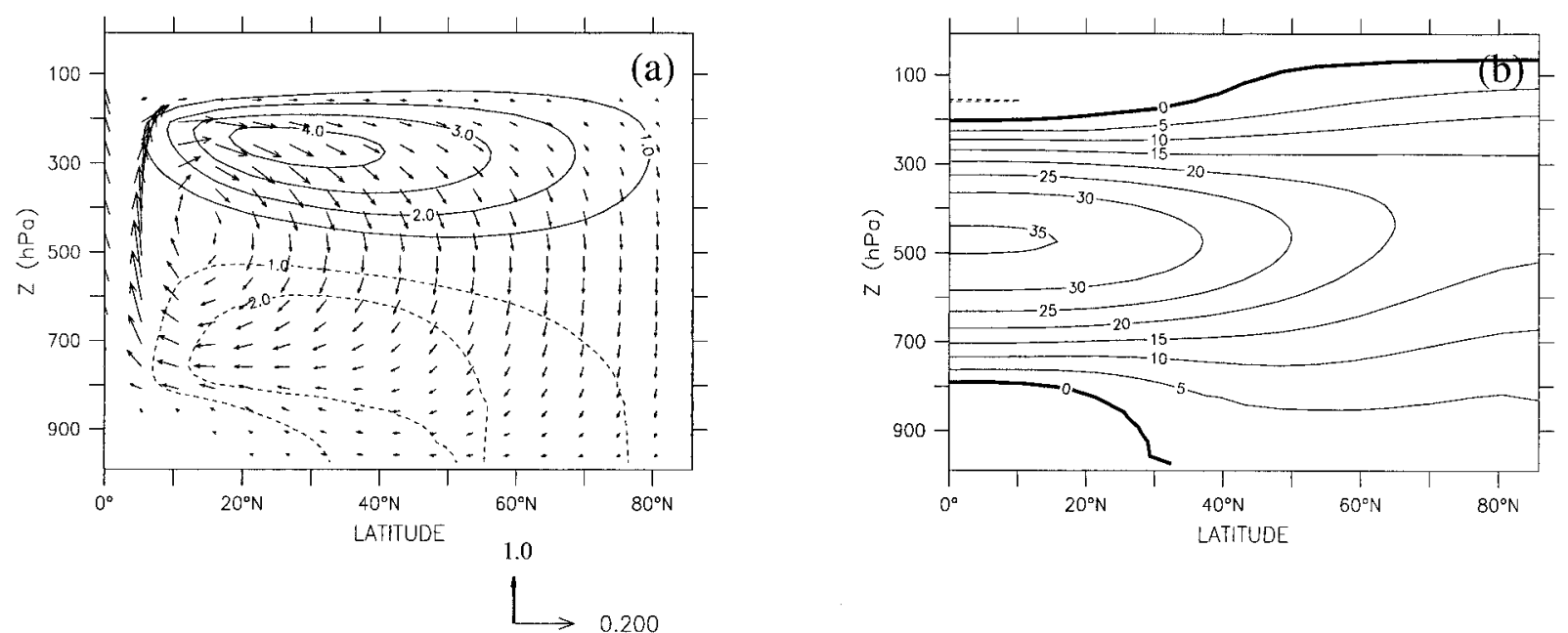

FIG. 13. Zonally averaged physical fields for the Rayleigh friction alone case. In (a) the vector field of meridional velocity (with unit $\mathrm{m}$ $\mathrm{s}^{-1}$ ) and vertical velocity $\left(-d p / d t\right.$ with unit $\mathrm{hPa}$ day $^{-1}$ ) is superposed on the contoured zonal velocity (with contour interval $1.0 \mathrm{~m} \mathrm{~s}^{-1}$ ). The zonal velocities and the vertical velocities are scaled separately and are shown under each panel, respectively. In (b), zonally averaged temperature perturbation field is plotted with contour interval of $5^{\circ} \mathrm{C}$.

The zonal overturning in the Rayleigh friction alone case is quite different from that in the Newtonian cooling alone case (cf. Figs. 14 and 7). In the Rayleigh friction alone case, the zonal overturning is zonally asymmetric about the heat source and the zonal wind anomaly in the upper troposphere is much thinner vertically but larger in strength than that in the lower troposphere in the Rayleigh friction alone case.

In Fig. 15, we plot vertical profiles of zonal velocity, vertical velocity, and temperature perturbation at different locations. Although a nonzero surface wind is seen at high latitudes, there is vanishingly small flow in the tropical region above and below the heating layers.

\section{3) Convergence OF LOW-LEVEl MASS AND MOISTURE}

The purpose of this paper is to examine the atmospheric response to an elevated large-scale steady heat source when the atmosphere is subjected to different types of damping. However, a related question is whether the forced flows provide enough moisture convergence to maintain the heat source.

The divergence at different altitudes and the vertically integrated mass convergence from surface to all levels below $400 \mathrm{hPa}$ for the two experiments are shown in Fig. 16. The convergence below the heat source in both cases is negligible. The transition altitude from convergence to divergence in the Rayleigh friction alone case is at about $400 \mathrm{hPa}$, which is much higher than that in the Newtonian cooling alone case. The convergence between 850 and $500 \mathrm{hPa}$ in the Newtonian cool- ing alone case is stronger than that in the Rayleigh friction alone case.

Importantly, the maximum vertically integrated mass convergence in both cases is smaller than $300 \mathrm{~kg} \mathrm{day}^{-1}$ $\mathrm{m}^{-2}$. Even if we assume that the mixing ratio of water vapor below $400 \mathrm{hPa}$ is everywhere $25 \mathrm{~g} \mathrm{~kg}^{-1}$ (which is about the saturation value of moisture at $30^{\circ} \mathrm{C}$ ), the total convergence of moisture from the surface to the top of atmosphere is still less than $7.5 \mathrm{~kg}_{\text {day }}{ }^{-1} \mathrm{~m}^{-2}$ (which corresponds to $7.5 \mathrm{~mm}$ day $^{-1}$ of precipitation) and is much less than the minimum moisture convergence required to maintain the imposed heating corresponding to $10 \mathrm{~mm}$ day $^{-1}$. If the moisture convergence is regarded as mainly a boundary layer process, then it is clear that the flows generated by a large-scale heat source with a base at $840 \mathrm{hPa}$ (or above) cannot provide enough moisture convergence to maintain that heat source.

\section{Role of a simple frictional boundary layer}

In the previous sections we found that when the damping is exclusively momentum damping (Rayleigh friction), the response of the atmosphere to a heat source localized in the vertical is constrained to the layers of the atmosphere that are heated; hence, there is no surface response unless the heating extends to the surface. In contrast, an elevated heat source does support a surface wind response in the case where the damping is exclusively thermodynamic (Newtonian cooling). Of course, this solution is unrealistic because any surface response must in nature experience momentum damping. In this 


\section{Rayleigh-Friction-Alone-Case}
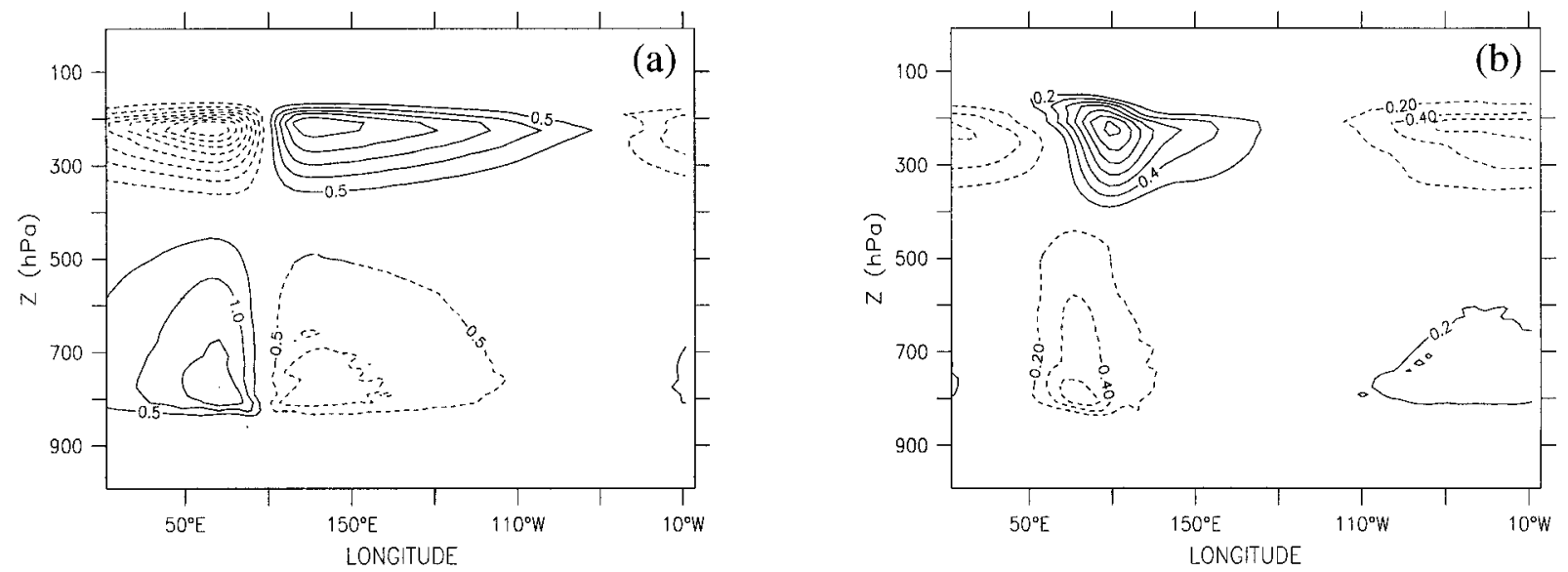

FIG. 14. Zonal velocity anomalies (i.e., the zonal wind minus the zonal mean zonal wind) at different latitude bands for the Rayleigh friction alone case. (a) The latitude band from $0^{\circ}$ to $10^{\circ} \mathrm{N}$, and (b) the latitude band $10^{\circ}$ to $20^{\circ} \mathrm{N}$. Contour intervals are 0.5 and $0.2 \mathrm{~m} \mathrm{~s}{ }^{-1}$ in (a) and (b), respectively. The zero contour lines are not shown.

section we perform one more integration in order to explore how the inclusion of a simple frictional boundary layer affects the surface response when there is also uniform thermal damping throughout the atmosphere. The heating and the Newtonian cooling rate are prescribed identically to those in section 3 ; the atmosphere above $850 \mathrm{hPa}$ is inviscid, and we have included a frictional boundary layer with a Rayleigh friction timescale of 2 days between 850 and $975 \mathrm{hPa}$ and 1 day below $975 \mathrm{hPa}$. Selected results from this experiment are shown in Fig. 17.

The panels in Fig. 17 show that including a simple representation of a boundary layer dramatically suppresses the surface circulation that was found in the Newtonian cooling alone case. Taken at face value the analytical and numerical results of sections 2 and 3 along with this experiment suggest that it is unlikely that an elevated heat source could generate a significant boundary layer circulation unless the base of heating is embedded well within the boundary. When the cloud base is at about $600 \mathrm{~m}$ above surface as observed by Mapes and Houze (1995), we cannot rule out the possibility of strong surface winds driven by the elevated heat source [see Fig. 4.9 of Wu (1998)]. Furthermore, since our simplification of the boundary layer only emphasizes the role of friction and does not include the role of strong vertical momentum mixing in the boundary layer, how a more realistic representation of the boundary layer can affect the results is not clear. Therefore, it remains to be seen to what extent this conclusion is sensitive to the formulations of friction and vertical mixing within the boundary layer and especially to the location of the base of the heating and its relationship to the top of the boundary layer.

\section{Discussion and conclusions}

We have examined the steady response of the tropical atmosphere to an isolated heat source in the Tropics using both an analytical method and a dry primitive equation model of the atmosphere. In the analytical part of the study, two special cases are examined: one has Newtonian cooling as the only dissipation to balance the energy input by the heating, while the other has Rayleigh friction and no thermal damping. To verify the analytical arguments, we carried out a series of numerical experiments.

The analytical results and the numerical results are consistent in showing that Rayleigh friction and Newtonian cooling play very different roles in the tropical atmosphere. When the characteristic meridional scale of the heat source is large enough (i.e., $>500 \mathrm{~km}$ ), Newtonian cooling homogenizes the atmospheric motion in the vertical, and a strong, vertically uniform wind is found below the bottom of the heat source. This result is also consistent with the results in Wu et al. (1999) in which a meridional modal decomposition method, outlined in the introduction of this paper, was used to exclusively investigate the Green's function response to an elevated large-scale heat source in the vertical in the presence of only Newtonian cooling. When Rayleigh friction serves as the only damping in the tropical atmosphere, there is not much interaction in the vertical between the forced and unforced atmospheric layers: all the energy put into a layer is dissipated within that layer. Hence, no surface wind is found unless the heat source reaches close to the surface. The horizontal structure of winds in this case is somewhat similar to the solution given by Gill (1980). 


\section{Rayleigh-Friction-Alone-Case}
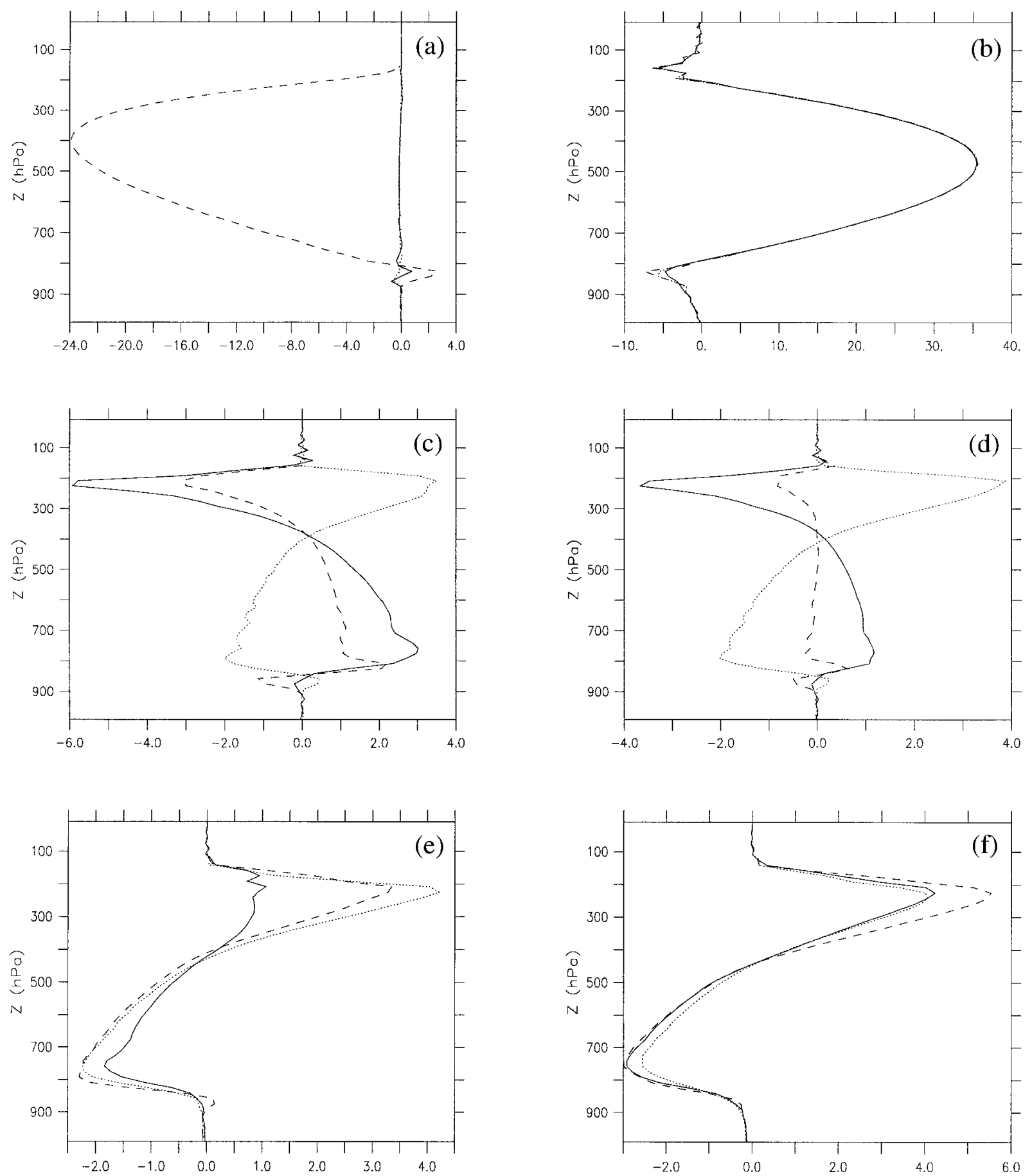

FIG. 15. Vertical profiles of the vertical velocities (a), the temperature perturbations (b) at the equator, and the zonal velocities at different latitudes [(c) at the equator, (d) at $5.4^{\circ} \mathrm{N}$, (e) at $11.7^{\circ} \mathrm{N}$; (f) at $16.1^{\circ} \mathrm{N}$ ] for the Rayleigh friction alone case. Solid lines are at $53.4^{\circ} \mathrm{E}$, dashed lines are at $92.8^{\circ} \mathrm{E}$, and dotted lines are at $132.2^{\circ} \mathrm{E}$. The units along the abscissa are $\mathrm{hPa}$ day ${ }^{-1},{ }^{\circ} \mathrm{C}$, and $\mathrm{m} \mathrm{s}^{-1}$ for the vertical velocity, the temperature perturbation, and the zonal velocity, respectively. 

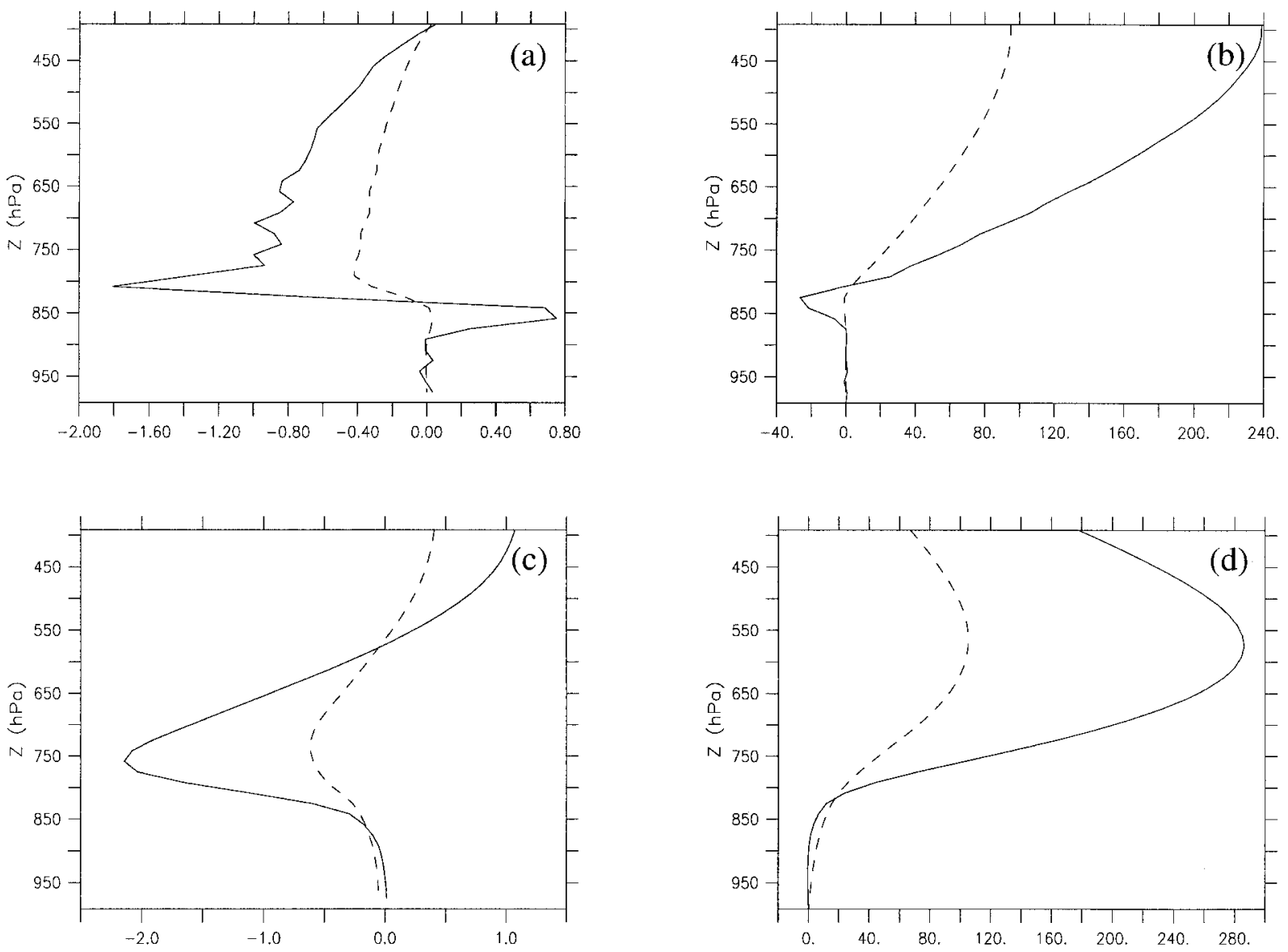

FIG. 16. The vertical profiles of the divergence $\left(10^{-6} \mathrm{~s}^{-1}\right)\left[(\mathrm{a})\right.$ and (c)] and the vertically integrated mass convergence $\left(\mathrm{kg} \mathrm{day}^{-1} \mathrm{~m}^{-2}\right)$ from the surface to different altitudes [(b) and (d)] for both the Rayleigh friction alone case [(a) and (b)] and the Newtonian cooling alone case [(c) and (d)]. Solid lines represent the quantities at the center of the heat source. Dashed lines represent the quantities averaged over a rectangle area from $11^{\circ} \mathrm{S}$ to $11^{\circ} \mathrm{N}$ and from $70^{\circ}$ to $115^{\circ} \mathrm{E}$.

From the analysis in section 2, we know that only when momentum damping is fairly strong can the signal in the Tropics propagate to the extratropics and a strong meridional overturning circulation will form. The strong momentum damping also causes the response to be vertically confined to the layer in which the heat source resides. Hence, the low-level flow in the deep Tropics (i.e., between $10^{\circ} \mathrm{N}$ and $10^{\circ} \mathrm{S}$ ) is not associated with a strong Hadley circulation driven by an elevated tropical heat source in the absence of background wind: it is possibly due to the surface pressure gradients generated by the hydrostatic adjustment to SST gradients. This conclusion is confirmed by our numerical results and is also quite consistent with the modeling results of Schneider and Lindzen (1977) and Battisti and Ovens (1995), who found that the surface winds in the deep Tropics are not associated with the thermally forced Hadley circulation.

The zonal overturning circulation (the Walker circulation) is identified in a narrow band centered about the equator in both the Rayleigh friction alone case and the Newtonian cooling alone case. However, the struc- ture of the zonal overturning cell is much different in these two cases. In the Newtonian cooling alone case, the zonal overturning cell (after removing the zonal means) is fairly symmetric about the heat source, while in the Rayleigh friction alone case, the overturning to the east of the heat source is wider than that to the west.

The numerical solution is also found for the case where Newtonian cooling acts uniformly in the vertical and Rayleigh friction is included in the lower atmosphere to mimic crudely the dissipation of momentum in the boundary layer. The introduction of the simple boundary layer dramatically reduces the surface circulation that was supported in the Newtonian cooling alone case. Together these results suggest that a significant surface circulation will unlikely be driven by an elevated heat source if it resides above the top of the boundary layer.

The vertically integrated mass convergence shows that the forced flow does not provide enough moisture convergence to maintain the heat source, suggesting that heating is not fueled enough by the moisture convergence associated with the thermally driven flows. There- 

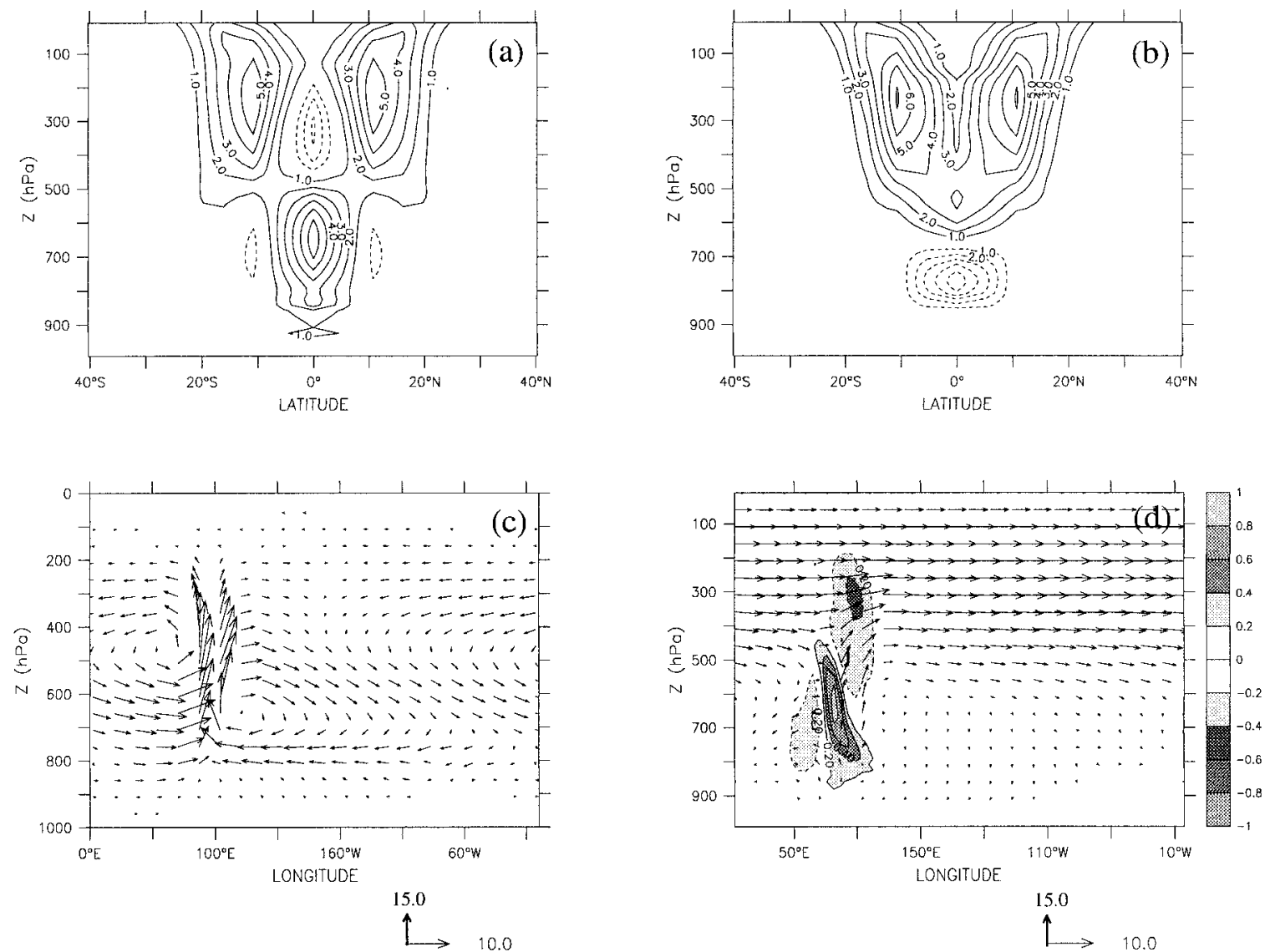

FIG. 17. Selected results from the case with uniform Newtonian cooling and a simple frictional boundary layer. (a) and (b) The zonal velocities at $53.4^{\circ}$ and $132.2^{\circ} \mathrm{E}$, respectively. Westerlies are represented by solid lines, and easterlies are represented by dashed lines. Contour interval is $1.0 \mathrm{~m} \mathrm{~s}^{-1}$ in both panels, and the zero contour lines are not shown. (c) and (d) the zonal overturning circulations at $0^{\circ}$ and $10.7^{\circ} \mathrm{N}$, respectively. The vector fields of zonal velocity (with unit $\mathrm{m} \mathrm{s}^{-1}$ ) and vertical velocity $\left(-d p / d t\right.$ with unit hPa day ${ }^{-1}$ ) are superposed on the contoured meridional velocity (with contour interval $0.2 \mathrm{~m} \mathrm{~s}^{-1}$ ). The zonal velocity and the vertical velocity are scaled separately and are shown under each panel.

fore, CISK cannot apply. Since our estimation of the moisture convergence is somewhat an upper limit and is not sensitively dependent on the base of the heating, we expect that the previous conclusion concerning the moisture convergence will hold even when a realistic boundary layer is included in the model.

Acknowledgments. We are grateful to E. Harrison and S. Hankin of NOAA/PMEL for their providing us with FERRET, a diagnostics and graphics program that has been intensively used in this study. The authors would also like to thank the anonymous reviewers for helpful and substantive comments. This work was supported by a grant from NOAA/Office of Global Programs to the Stanley P. Hayes Center of JISAO at the University of Washington.

\section{REFERENCES}

Bantzer, C. H., and J. M. Wallace, 1996: Intraseasonal variability in tropical mean temperature and precipitation and their relation to the tropical 40-50 day oscillation. J. Atmos. Sci., 53, 3032-3045.
Battisti, D. S., and D. Ovens, 1995: The dependence of the low-level equatorial easterly jet on Hadley and Walker circulations. $J$. Atmos. Sci., 52, 3911-3931.

— A. C. Hirst, and E. S. Sarachik, 1989: Instability and predictability in coupled atmosphere-ocean models. Philos. Trans. Roy. Soc. London, A329, 237-247.

Deser, C., and J. M. Wallace, 1987: El Niño events and their relationship to Southern Oscillation phenomenon. J. Geophys. Res., 92, 14 189-14 196.

_ and - 1990: Large-scale atmospheric circulation feature of warm and cold episodes in the tropical Pacific. J. Climate, 3, $1254-1281$.

Flattery, T. W., 1967: Hough functions. Ph.D. dissertation, Department of Geophysical Sciences, University of Chicago, 168 pp.

Geisler, J. E., 1981: A linear model of the Walker circulation. J. Atmos. Sci., 38, 1390-1400.

Gill, A. E., 1980: Some simple solutions for heat induced tropical circulations. Quart. J. Roy. Meteor. Soc., 106, 447-462.

Graham, N. E., T. P. Barnett, R. Wilde, M. Ponater, and S. Shubert, 1994: On the roles of tropical and midlatitude SST in forcing interannual to interdecadal variability in the winter Northern Hemisphere circulation. J. Climate, 7, 1416-1441.

Holton, J. R., 1992: An Introduction to Dynamic Meteorology. Academic Press, $511 \mathrm{pp}$.

, and D. E. Colton, 1972: A diagnostic study of the vorticity 
balance at $200 \mathrm{mb}$ in the Tropics during the northern summer. J. Atmos. Sci., 29, 1124-1128.

Lamb, H., 1932: Hydrodynamics. Cambridge University Press, 738 pp.

Lau, N. C., and M. J. Nath, 1994: A modeling study of the relative roles of tropical and extratropical SST in the variability of the global atmosphere-ocean system. J. Climate, 7, 1184-1207.

Lindzen, R. S., 1967: Planetary waves on beta-planes. Mon. Wea. Rev., 95, 441-451.

- 1968: Vertically propagating waves in an atmosphere with Newtonian cooling inversely proportional to density. Can. J. Phys. 46, 1835-1840.

_ 1990: Dynamics in Atmospheric Physics. Cambridge University Press, $310 \mathrm{pp}$.

- , and S. Nigam, 1987: On the role of sea surface temperature gradients in forcing low-level winds and convergence in the tropics. J. Atmos. Sci., 44, 2418-2436.

—, E. S. Batten, and J. -W. Kim, 1968: Oscillations in atmospheres with tops. Mon. Wea. Rev., 96, 133-140.

Mapes, B. E., and R. A. Houze, 1995: Diabatic divergence profiles in western Pacific mesoscale convective system. J. Atmos. Sci., 52, 1807-1828.

Miller, R. L., and R. S. Lindzen, 1992: Organization of rainfall by an unstable jet with an application to African waves. J. Atmos. Sci., 49, 1523-1540.

Mitchell, T. P., and J. M. Wallace, 1996: ENSO seasonality: 195078 versus 1979-92. J. Climate, 9, 3149-3161.

Philander, S. G., 1990: El Niño, La Niña, and the Southern Oscillation. Academic Press, 293 pp.

Rasmusson, E. M., and T. H. Carpenter, 1982: Variation in tropical sea surface temperature and surface wind fields associated with the Southern Oscillation/El Niño. Mon. Wea. Rev., 110, 354384.
Reed, R. J., and E. E. Recker, 1971: The vorticity budget of synopticscale wave disturbances in the equatorial western Pacific. J. Atmos. Sci., 28, 1117-1133.

Robertson, A. W., and C. Frankignoul, 1990: The tropical circulation: Simple model versus general circulation model. Quart. J. Roy. Meteor. Soc., 116, 69-87.

Saravanan, R., and J. C. McWilliams, 1995: Multiple equilibria, natural variability, and climate transitions in an idealized oceanatmosphere model. J. Climate, 8, 2296-2323.

Schneider, E. K., 1977: Axially symmetric steady-state models of the basic state for instability and climate studies. Part II: Nonlinear circulations. J. Atmos. Sci., 34, 263-279.

, and R. S. Lindzen, 1977: Axially symmetric steady-state models of the basic state for instability and climate studies. Part I: Linearized circulations. J. Atmos. Sci., 34, 263-279.

Wallace, J. M., 1971: Spectral studies of tropospheric wave disturbances in the tropical western Pacific. Rev. Geophys. Space Phys., 9, 557-612.

Webster, P. J., 1972: Response of tropical atmosphere to local steady forcing. Mon. Wea. Rev., 100, 518-541.

Wu, Z., 1998: Thermally driven surface winds in the tropics. Ph.D. dissertation, Department of Atmospheric Sciences, University of Washington, $178 \mathrm{pp}$.

- E. S. Sarachik, and D. S. Battisti, 1999: Thermally forced planetary waves on an equatorial beta plane. J. Atmos. Sci., 56, 2029-2037.

cture of convective heating and the three-dimensional structure of the forced circulation on an equatorial beta plane. J. Atmos. Sci., 57, 2169-2187.

Yulaeva, E., and J. M. Wallace, 1994: The signature of ENSO in global temperature and precipitation fields derived from the Microwave Sounding Unit. J. Climate, 7, 1719-1736.

Zebiak, S. E., 1986: Atmospheric convergence feedback in a simple model for El Niño. Mon. Wea. Rev., 114, 1263-1271. 